\title{
Cavity-flow wall effects and correction rules
}

\section{By T. YAO-TSU WU, ARTHUR K. WHITNEY AND CHRISTOPHER BRENNEN}

California Institute of Technology

(Received 18 December 1970)

This paper is intended to evaluate the wall effects in the pure-drag case of plane cavity flow past an arbitrary body held in a closed tunnel, and to establish an accurate correction rule. The three theoretical models in common use, namely, the open-wake, Riabouchinsky and re-entrant-jet models, are employed to provide solutions in the form of some functional equations. From these theoretical solutions several different rules for the correction of wall effects are derived for symmetric wedges. These simple correction rules are found to be accurate, as compared with their corresponding exact numerical solutions, for all wedge angles and for small to moderate 'tunnel-spacing ratio' (the ratio of body frontal width to tunnel spacing). According to these correction rules, conversion of a drag coefficient, measured experimentally in a closed tunnel, to the corresponding unbounded flow case requires only the data of the conventional cavitation number and the tunnel-spacing ratio if based on the open-wake model, though using the Riabouchinsky model it requires an additional measurement of the minimum pressure along the tunnel wall.

The numerical results for symmetric wedges show that the wall effects invariably result in a lower drag coefficient than in an unbounded flow at the same cavitation number, and that this percentage drag reduction increases with decreasing: wedge angle and/or with decreasing tunnel spacing relative to the body frontal width. This indicates that the wall effects are generally more significant for thinner bodies in cavity flows, and they become exceedingly small for sufficiently blunt bodies. Physical explanations for these remarkable features of cavity-flow wall effects are sought; they are supported by the present experimental investigation of the pressure distribution on the wetted body surface as the flow parameters are varied. It is also found that the theoretical drag coefficient based on the Riabouchinsky model is smaller than that predicted by the open-wake model, all the flow parameters being equal, except when the flow approaches the choked state (with the cavity becoming infinitely long in a closed tunnel), which is the limiting case common to all theoretical models. This difference between the two flow models becomes especially pronounced for smaller wedge angles, shorter cavities, and with tunnel walls farther apart.

In order to gauge the degree of accuracy of these theoretical models in approximating the real flows, and to ascertain the validity of the correction rules, a series of definitive experiments was carefully designed to complement the theory, and then carried out in a high-speed water tunnel. The measurements on a series 
of fully cavitating wedges at zero incidence suggest that, of the theoretical models, that due to Riabouchinsky is superior throughout the range tested. The accuracy of the correction rule based on that model has also been firmly established. Although the experimental investigation has been limited to symmetric wedges only, this correction rule (equations (85), (86) of the text) is expected to possess a general validity, at least for symmetric bodies without too large curvatures, since the geometry of the body profile is only implicitly involved in the correction formula. This experimental study is perhaps one of a very few with the particular objective of scrutinizing various theoretical cavity-flow models.

\section{Introduction}

The wall effects in cavity flows occurring in water tunnels have been recognized to be considerably more appreciable and more difficult to determine than those in the wind tunnel (or water tunnel) tests of non-separated (or noncavitating) flows past an obstacle. The main difficulty may be attributed primarily to the presence of a cavity boundary which renders this free-surface flow problem intrinsically non-linear in the sense that the configuration of the body-cavity system will change as the wall-spacing or the cavitation number varies, whereas in non-separated or non-cavitating flows the body shape always remains the same. Another complication arises from the fact that in experiments the cavitation number cannot be predicted in terms of known free-stream conditions, but still must be measured, since the cavity pressure is a manifestation of the entire flow, and this flow parameter may change as the body scale and/or the wall spacing is varied. Partly for these reasons, the state of knowledge about the wall effects in cavity flows has not been as well developed as its counterpart in wind tunnel experimental techniques. (For a general review of the latter see Pope (1958) and the cited references.) In fact, there does not seem to exist an accurate formula or rule for the tunnel-wall correction of cavity flows in general. The principal object of this study is to investigate the effects of relevant flow parameters, and to establish a simple wall-correction rule for cavity flows.

The physical flow boundaries in the test section of water tunnels may be classified in three different types: (a) rigid walls of closed tunnels, $(b)$ a free surface of constant pressure if the tunnel uses a free jet, and (c) a combination of free and solid surfaces, such as in a partly bounded jet tunnel, or in a horizontal free-surface channel with rigid bottom and sides. The presence of these flow boundaries will introduce several significant effects: (i) In dealing with the potential portion of the flow, these flow boundaries will impose a condition either on the flow direction at rigid walls or on the pressure at a free surface. In most cases, they will produce extraneous forces and modifications of cavity shape that should be corrected if the experimental results are to represent the corresponding unbounded flows. (ii) In closed tunnels, the boundary layer built up at the solid tunnel walls in a long straight test section may effectively reduce the tunnel area, and generate a longitudinal pressure gradient in the working section, giving rise to an additional drag force known as the 'horizontal buoyancy'. It is usually small in closed test sections, and can be compensated for by having slightly 
diverging walls, or adjustable walls. This viseous effect may also produce, near the intersection of a two-dimensional model and the tunnel walls, a secondary flow, which will further change the primary flow field. (iii) The lateral constraint in closed test-sections will result in a higher velocity outside the boundary layer, and hence a greater skin friction at the wetted body surface. However, when the Reynolds number $R e$ is large, as is generally the case in practice, the boundary-layer-induced pressure field is quite small (of $O\left(R e^{-\frac{1}{2}}\right)$ in drag, and of $O\left(R e^{-\frac{1}{2}} \log R e\right)$ in lifting flows). (iv) The lateral constraint also affects the spreading of the viscous wake behind the cavity, an effect known as the "wake blocking'. This effect increases the drag of a model in a closed wind tunnel (see Pope 1958; Allen \& Vincenti 1944), and is usually negligible in open jets. For cavity flows inside a closed water-tunnel, however, this 'wake-blocking' effect is usually less significant than the effect on the change in cavity shape and in cavitation number (both of the latter being already included in (i) above). (v) In the case where cavity-boundary detachment from a curved body (such as a circular cylinder) is smooth (characterized by the curvature of the cavity boundary being finite at detachment), the point of detachment will depend on both the cavitation number and the wall spacing. In such cases, correlation between the experimental results and the unbounded flow would be even more complicated. In the present work, effect (i) will be investigated for the pure-drag flows, so that this primary effect can be clarified first. The same effect in the case of lifting flows will be discussed in a future paper. Effect (ii) can be evaluated with some modifications of the present formulation by incorporating the method for wind-tunnel tests of Allen \& Vincenti (1944) or that of Glauert (1933). Effect (iii) can be estimated by taking the boundary layer into account, as will be done in $\S 8$, when the experimental data are analyzed. The secondary effect (iv) is expected to be even less significant than in the wind-tunnel case, since the wake would be further downstream, and will not be pursued here. The effect $(v)$ is, however, beyond the scope of the present study.

In the past, a few special problems of wall effects have been discussed. The choked cavity flow (i.e. when the cavity is infinitely long in a closed tunnel or in a free jet) attracted early attention due to its relative simplicity. Birkhoff, Plesset \& Simmons (1950) treated the non-lifting case of a wedge. Suppose a symmetric body of frontal area $A$, placed centrally in a tunnel, experiences a drag $D$ in a choked cavity flow with upstream velocity $U$ and pressure $p_{\infty}$. Define two drag coefficients,

$$
C_{D}=D / \frac{1}{2} \rho U^{2} A, \quad C_{D}^{\prime}=D / \frac{1}{2} \rho q_{c}^{2} A,
$$

where $q_{c}$ is the constant velocity at the cavity boundary. For a flat plate set broadwise to flow, Birkhoff, Plesset \& Simmons (1950) showed theoretically that the conventional drag coefficient $C_{D}$ depends strongly on the spacing of the channel walls, whereas $C_{D}^{\prime}$ is almost insensitive to it. When the test section is a free jet, both $C_{D}$ and $C_{D}^{\prime}$ are found to be insensitive to the jet width (down to the body width); this may be attributed to the fact that for an infinite cavity in a free jet the two velocities $U$ and $q_{c}$ are equal. These results were predicted earlier by 
Valcovici (1913) using methods suggested by Prandtl. Now, by Bernoulli's theorem,

$$
p+\frac{1}{2} \rho q^{2}=p_{\infty}+\frac{1}{2} \rho U^{2}=p_{c}+\frac{1}{2} \rho q_{c}^{2}=p_{b}+\frac{1}{2} \rho V^{2}=p_{s},
$$

where $p_{c}$ is the cavity pressure, $p_{s}$ the stagnation pressure, and $p_{b}$ is the pressure associated with a third reference velocity $V$ (to be used later in this study). Then

$$
C_{D}=\left(q_{c} / U\right)^{2} C_{D}^{\prime}=(1+\sigma) C_{D}^{\prime}
$$

where $\sigma$ is the conventional cavitation number,

$$
\sigma=\left(p_{\infty}-p_{c}\right) / \frac{1}{2} \rho U^{2}=\left(q_{c} / U\right)^{2}-1 .
$$

Now, since $C_{D}^{\prime}$ is nearly constant (equal to 0.88 for the flat plate set normal to stream), and the factor $(1+\sigma)$ gives an accurate dependence of $C_{D}$ on $\sigma$ for a plate in an unbounded flow (for $0<\sigma<1$, see e.g. Gilbarg 1960; Wu 1968), the above relationship (3) has led Birkhoff (1950) to assert the stronger 'principle of stability of the pressure coefficient': that for an obstacle of given shape in a water tunnel (or jet) the pressure coefficient,

$$
\begin{gathered}
C_{p}^{\prime}=\left(p-p_{c}\right) / \frac{1}{2} \rho q_{c}^{2}=1-\left(\frac{q}{q}\right)^{2}, \\
C_{p}=\left(p-p_{\infty}\right) / \frac{1}{2} \rho U^{2}=1-\left(\frac{q}{U}\right)^{2},
\end{gathered}
$$

rather than

is insensitive to the presence of walls and changes in the cavitation number $\sigma$. This principle, elegant and useful as it may be for blunt bodies, unfortunately does not possess a general validity. In fact, as the result of this work will show later, the wall effects in terms of either $C_{D}$ or $C_{D}^{\prime}$ are rather insignificant for blunt bodies. For symmetric wedges, the wall effect on $C_{D}$ increases with decreasing wedge angle and the effect on $C_{D}^{\prime}$ is actually more pronounced.

In the general case of unchoked cavity flows in a bounded stream, various attempts have been made to apply different theoretical flow models in some simple cases. The Riabouchinsky model was adopted by Cisotti (1922) for cavity flow past a plate in a tunnel, by Simmons (1948) and by Birkhoff et al. (1952) for a plate either in a tunnel or in a free jet. The re-entrant jet model was used by Gurevich (1953) for a wedge in a tunnel. The open-wake model of Joukowsky \& Roshko, which turns out to be the simplest in numerical detail though it has not, to the author's knowledge, been employed before, is considered here along with the other models in formulating the general problem of an arbitrary body in symmetrical tunnel flow.

The problem of wall effects on lifting cavity flows is more complicated, owing to the lack of a basic symmetry. The case of choked flow past an inclined flat plate within a straight tunnel has been investigated by Ai (1966). A linearized theory for choked flows past vented or cavitating hydrofoils has been developed by Fabula (1964). The wall effect on the force coefficients of an inclined flat plate with a finite cavity have been evaluated by Wu, Whitney \& Lin (1969), based on the open-wake model. A general discussion of the lifting cavity flows will be presented in a future paper. 
An entirely different approach to this problem for a thin body at small incidence is based on the linearized cavity-flow theory. This linearized theory has been developed for wall-effect problems by Cohen \& Di Prima (1958), Cohen \& Gilbert (1957), Cohen, Sutherland \& Tu (1957), and by Fabula (1964). Some comparison between the non-linear and linear theories will be made in this study (see $\S 8$ ).

Recently, Brennen (1969b) adopted the Riabouchinsky model to evaluate the wall effect for axisymmetric flows with a finite cavity past a disk and a sphere; he also obtained some new experimental results. In Brennen's relaxation method, the flow is bounded laterally by a concentric cylinder of various sizes, down to the smallest that produces the choked flow at one cavitation number, and the unbounded flow case is reached by extrapolation. The numerical results thus also furnish useful information about the wall effect in axisymmetric cavity flows. This problem has been discussed previously by Armstrong \& Tadman (1953), and by Campbell \& Thomas (1956) for the variation of the cavity dimensions.

A clear understanding of the wall effects in wake or cavity flows is essential to interpret correctly the experimental results. Grove et al. (1964) investigated experimentally the steady separated flow past a circular cylinder (of diameter $d$ ) in an oil tunnel (of spacing $h$ ) with the Reynolds number going up to about 300 . For $d / h=0 \cdot 05$, the base pressure coefficient was found to reach the asymptote -0.45 for $R e>25$ (up to $R e=177$ ). It was then conjectured that the pressure profile for $d / h=0.05$ had already reached the limiting form as $d / h \rightarrow 0$ (the unbounded flow case). However, this final extrapolation is misleading, since a simple estimate (e.g. by using (10) below) indicates that the flow state at hand is right in the vicinity of the choked flow limit.

Experimental studies designed to investigate the wall effects in cavity flows have received increasing attention recently. A review of these activities has been given by Morgan (1966). Dobay (1967) investigated experimentally the blockage effects on axisymmetric cavity flows past circular disks, set normal to the flow, and showed that choking occurred even with the disk-to-tunnel diameter ratio reduced to $\frac{1}{36}$. Similar findings have been reported by Barr (1966).

In another experimental endeavour, Meijer (1967) investigated the tunnelwall effect on a cavitating hydrofoil with a flap. An empirical method for correcting the wall effect was chosen, which is based on a different pressure coefficient $C_{p}^{\prime \prime}$ and cavitation number $\sigma^{\prime \prime}$, defined as (see (2))

$C_{p}^{\prime \prime}=\left(p-p_{b}\right) / \frac{1}{2} \rho V^{2}=1-\left(\frac{q}{V}\right)^{2}, \quad \sigma^{\prime \prime}=\left(p_{b}-p_{c}\right) / \frac{1}{2} \rho V^{2}, \quad C_{D}^{\prime \prime}\left(\sigma^{\prime \prime}\right)=D / \frac{1}{2} \rho V^{2} A$,

where $p_{b}$ is the minimum pressure and $V$ is the corresponding maximum velocity on the tunnel wall. In light of the present theoretical result and more definitive experimental verifications, Meijer's rule appears generally to over-correct the cavity-flow wall effects, as will be shown later. This defect has also been discovered, independently, by Meijer (1969), who proposed a modified empirical method.

Finally, it is of interest to point out the different trends between the wall effects in non-separated, non-cavitating flows and those in cavity flows. In closed wind tunnels, the lateral constraint and body thickness generally result 
in an increase of flow velocity and hence dynamic pressure, thus increasing lift, drag, and moment coefficients at a given angle of attack (see e.g. Pope 1958). In contrast, the general trend of the wall effect on cavity flows in closed tunnels have been found to decrease the drag and lift coefficients at prescribed cavitation number and incidence. These opposite trends may seem at first glance puzzling, particularly to those experienced with wind-tunnel testings. Actually, the lateral constraint in the presence of a cavity still results in an increase of flow velocity, and hence a decrease of the pressure over the wetted surface of the body, consequently decreasing all the forces if referred to the same cavitation number. Furthermore, this increase in flow velocity at the cavity boundary will cause the cavity pressure to be somewhat lower, and hence the cavitation number somewhat higher than in an unbounded flow with the same free stream condition. These two effects therefore reinforce each other such that the curve of $C_{D} v s . \sigma$ lies below the corresponding curve for unbounded flow.

\section{Theoretical models for inviscid cavity flows: momentum considera- tions}

The theoretical models in common use for treating steady inviscid cavity flows have been known to predict hydrodynamic forces on blunt obstacles with differences so small that they are usually beyond the limit of experimental accuracy (see e.g. Gilbarg 1961). It is also known that these models, when applied to unsteady cavity-flow problems, yield appreciably different results (see Wang \& $\mathrm{Wu}$ 1963). Since the real-fluid effects in the wake are approximated by different artifices in different models and the cavity drag is distributed, according to these artifices, at different rates in different regions, it is of interest to examine these models in the presence of strong wall effects. This will be done in two steps. First, the over-all features will be studied in the light of simple momentum consideration. The rest will be complemented by the detailed analysis. The final results for thin obstacles exhibit appreciable differences between the three models adopted. This theoretical result has helped plan the experimental investigations for a crucial evaluation of the theoretical models, as will be discussed in $\S 8$.

Before we deal with the inviscid cavity- or wake-flow models, let us consider a typical viscous, incompressible flow past a blunt body which is propelled along the axis of an infinitely long straight tunnel by an external force. The body moves at a sufficiently high Reynolds number $R e$ so that a recirculating near wake (or a finite cavity in a two-phase flow) is established. For simplicity, the additional viscous effect due to the boundary layer built up along the tunnel walls will be avoided by assuming that the walls can be made to move with an appropriate tangential velocity so as to eliminate the boundary layer altogether. Then, with respect to the body frame, the upstream velocity will be $U$, say, and the pressure $p_{\infty}$ (see figure $1(a)$ ). At large distances downstream (say for $x \geqslant \rho U^{2} A_{T}^{\frac{3}{2}} / D$, where $A_{T}$ is the cross-sectional area of the tunnel and $D$ is the drag of the body), where the turbulent far wake has spread uniformly across the channel, or even after the turbulence has dissipated and degenerated into a laminar flow, the 
mean velocity will again be uniform and equal to $U$ on account of the continuity. But the pressure, after full recovery of the kinetic energy, will be $p_{b}$ say, which must be less than $p_{\infty}$, by the simple momentum consideration,

$$
D=\left(p_{\infty}-p_{b}\right) A_{T}, \quad \text { or } \quad C_{p}^{-}=\left(p_{\infty}-p_{b}\right) / \frac{1}{2} \rho U^{2}=\frac{A}{A_{T}} C_{D}
$$

$A$ being the body frontal area, and $C_{D}$ being defined by (1). Thus, the wall effect here reduces the momentum defect to zero, and gives rise to an under-pressure in the downstream. This under-pressure coefficient $C_{p}^{-}$diminishes in proportion to the ratio $A / A_{T}$ as $A / A_{T} \rightarrow 0$, since $C_{D}$ must remain finite. (In plane flows, $A_{T}$ is replaced by the tunnel wall spacing $h$, and $A$ by the body frontal width $l$ ).

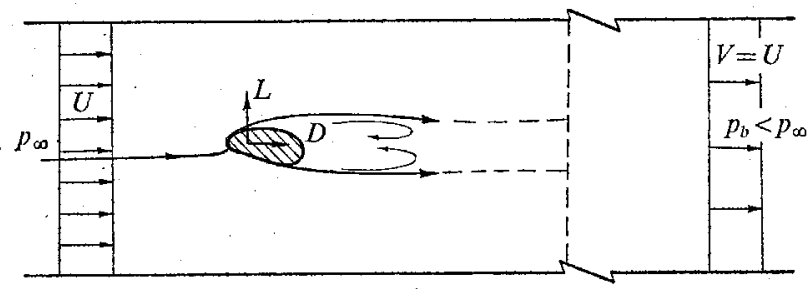

(a)

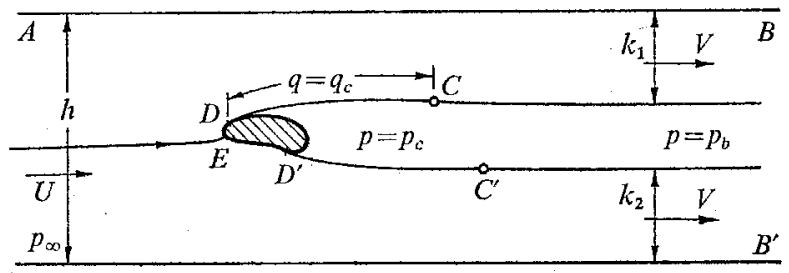

(b)

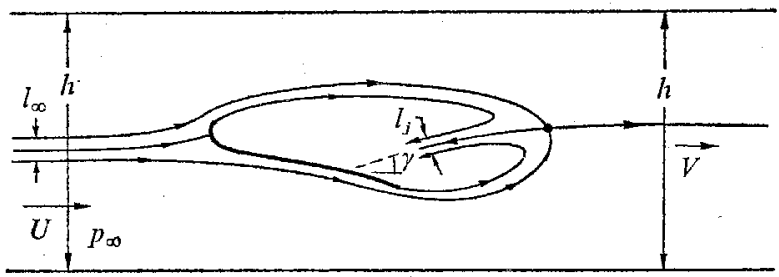

(c)

Fugure 1. Momentum considerations for cavity and wake flows.

We now turn to consider the cavity-flow models for an arbitrary body placed in a straight tunnel, with a finite cavity formation. Although they have been applied exclusively to plane flow analysis, the following momentum theorems are also valid for the three-dimensional case provided the relevant lengths are appropriately converted to their corresponding areas. 


\subsection{Open wake model}

According to this model, which is due to Joukowsky (1890), Roshko (1954), and Eppler (1954), and was modified by $\mathrm{Wu}$ (1962), the dividing streamline is tangential to the body surface $\left(E D\right.$ and $E D^{\prime}$ in the cross-sectional view of figure $1(b)$ ), detaches from the body at $D$ and $D^{\prime}$ to form a cavity boundary $D C$ and $D^{\prime} C^{\prime}$ over which the flow speed assumes a prescribed constant value $q_{c}$ (pressure $p_{c}$ ), and then proceeds downstream along $C B$ and $C^{\prime} B^{\prime}$, becoming asymptotically parallel to the walls so that the flow cross section approaches, $k\left(=k_{1}+k_{2}\right.$ in figure $\left.1(b)\right)$, the velocity becomes $V$, and the pressure $p_{b}$. The shape of $C B$ and $C^{\prime} B^{\prime}$ is such that this variable pressure part of the boundary makes no net contribution to the force on the body. Both $V$ and $k$ are unknown $a$ priori, but must satisfy the continuity equation

$$
U h=V k \text {. }
$$

The momentum consideration for the longitudinal component of the flow gives

$$
D=\left(p_{\infty}-p_{c}\right) h-\left(p_{b}-p_{c}\right) k+\rho U^{2} h-\rho V^{2} k,
$$

which becomes, upon using (2) and (8),

$$
C_{D}=D / \frac{1}{2} \rho U^{2} l=\frac{h}{l}\left(\frac{V}{U}-1\right)\left(\frac{q_{c}^{2}}{U V}-1\right)
$$

where $l$ denotes the frontal body width for plane flows (or the body frontal area in three-dimensional flows).

It is of particular significance to consider the choked flow limit (when the cavity becomes infinitely long and $V$ increases towards $q_{c}$ ). Let the corresponding limit of $U, C_{D}$ and the cavitation number $\sigma$, with $h / l$ and $q_{c}$ held fixed, be denoted by $U_{*}, C_{D_{*}}$ and $\sigma_{*}$ respectively, then

$$
C_{D_{*}}=\frac{1}{\lambda}\left(\frac{q_{c}}{U_{*}}-1\right)^{2}=\frac{1}{\lambda}\left[\left(1+\sigma_{*}\right)^{\frac{1}{2}}-1\right]^{2} \quad(\lambda=l / h) .
$$

$\sigma_{*}$ is called the choked cavitation number. From (10) it follows that

$$
\sigma_{*}=2\left(\lambda C_{D_{*}}\right)^{\frac{1}{2}}+\left(\lambda C_{D_{*}}\right)>2\left(\lambda C_{D_{*}}\right)^{\frac{1}{2}} \text {. }
$$

It is to be noted that $\sigma_{*}$ provides a lower limit of $\sigma$ below which the flow is physically not feasible, and that the right-hand side of (11) is a fairly accurate estimate of $\sigma_{*}$ for small $\lambda$. Thus, to achieve $\sigma=0 \cdot 1$, we must have $h / l>400$ if $C_{D_{*}} \simeq 1$.

Another point of interest is that the choked flow drag coefficient can be expressed in terms of the geometry by using (8). Since $U_{*} h=q_{c} k,(10)$ and (3) qecome

$$
C_{D_{*}}=\frac{1}{\lambda}\left(\frac{h}{k}-1\right)^{2}, \quad C_{D_{*}}^{\prime}=\left(\frac{q_{c}}{U_{*}}\right)^{-2} C_{D_{*}}=\frac{1}{\lambda}\left(1-\frac{k}{h}\right)^{2} .
$$

In the case of bluff bodies $C_{D_{*}}^{\prime}$ is insensitive to $\lambda$. Then

$$
k / h=1-\left(\lambda C_{D_{*}}^{\prime}\right)^{\frac{1}{2}}
$$

gives an estimate of $k / h$ in terms of $\lambda$. 
When the cavity is finite in length, we must have $U<V<q_{c}$. For sufficiently small $\lambda, V \sim U$ (see (9)) and the under-pressure coefficient at the downstream end becomes

$$
\dot{C}_{p}^{-}=\left(p_{\infty}-p_{b}\right) / \frac{1}{2} \rho U^{2}=(V / U)^{2}-1 \simeq \lambda\left(2 C_{D} / \sigma\right) \quad\left(\sigma>\sigma_{*}\right) ;
$$

thus $C_{p}^{-}$is proportional to $\lambda$, as in the case of (7), which is based on the viscous flow argument. However, very close to the choked flow state, (9),

$$
C_{p}^{-} \simeq\left(\lambda C_{D_{*}}\right)^{\frac{1}{2}} \quad\left(\sigma \simeq \sigma_{*}\right)
$$

which decreases much slower with decreasing $\lambda$.

\subsection{Re-entrant jet model}

A description of this model, which has been attributed independently to Kreisel, Gilbarg \& Efros, can be found in Gilbarg (1960). As shown in figure 1(c), let the downstream uniform state be characterized by velocity $V$ and pressure $p_{b}$. The jet flows upstream through the cavity into a second Riemann sheet, approaching a uniform state of velocity $q_{c}$, cross-sectional area $l_{j}$ and inclination $\gamma$ to the upstream flow direction. Then the continuity condition requires

$$
(U-V) h=q_{c} l_{j}
$$

In contrast to the open-wake model, we now have $V<U$, and hence $p_{b}>p_{\infty}$ (an over-pressure at the downstream!) as the momentum defect is carried off by the jet. Since the longitudinal momentum flux in the jet is $\left(-\rho q_{c} \cos \gamma\right)\left(q_{c} l_{j}\right)$, we now have the momentum equation,

$$
D=\left(p_{\infty}-p_{c}\right) h-\left(p_{b}-p_{c}\right) h+\rho\left(U^{2}-V^{2}\right) h+\rho l_{j} q_{c}^{2} \cos \gamma,
$$

which is reduced, upon using (16) and (2), to

$$
C_{D}=D / \frac{1}{2} \rho U^{2} l=\frac{h}{l}\left(1-\frac{V}{U}\right)\left(1+\frac{V}{U}+2 \frac{q_{c}}{U} \cos \gamma\right) .
$$

The choked flow state cannot readily be derived from the above formulas (it can however be deduced from the analysis later). Nevertheless, the limit must evidently be the same as (10) and (11) by virtue of the momentum consideration. In the non-choked condition, the over-pressure at the downstream end is

$$
C_{p}^{+}=\left(p_{b}-p_{\infty}\right) / \frac{1}{2} \rho U^{2}=1-\left(\frac{V}{U}\right)^{2} \sim\left(\frac{l}{h}\right) C_{D} /\left(1+\frac{q_{c}}{U} \cos \gamma\right)
$$

\subsection{Riabouchinsky model}

The main features of this model are shown in the typical case to be discussed in $\S 4$. Since there is no more than one distinct uniform flow state, the simple momentum argument cannot be employed to determine the drag, despite the fact that the choked flow state must agree with the other models. On the other hand, this model has an advantage of providing a point (point $B$ in figure $6, \S 4$ ) at which the velocity is maximum, and pressure minimum on the tunnel wall. This velocity is to be used in calculating $C_{p}^{\prime \prime}$ as defined in (6). 
In the remainder of this paper, we consider the pure-drag cavity flow past a symmetric body of arbitrary shape, placed symmetrically in a straight tunnel of width $h$, with a finite cavity attached to the body. The characteristic Reynolds number and the Froude number based on the body dimension are both assumed to be so large that the viscous and gravitational effects may be neglected. The solution will be determined by using three different flow models.

\section{Open-wake model}

This semi-infinite open-wake model has already been described in $\S 2$. As shown in figure 2, the boundaries $C B$ and $C^{\prime} B^{\prime}$ of the variable pressure part of the open wake now become straight and parallel to the $x$ axis by virtue of the flow symmetry. The flow region in the strip $|\psi| \leqslant \psi_{1}=\frac{1}{2} U h$ of the complex potential

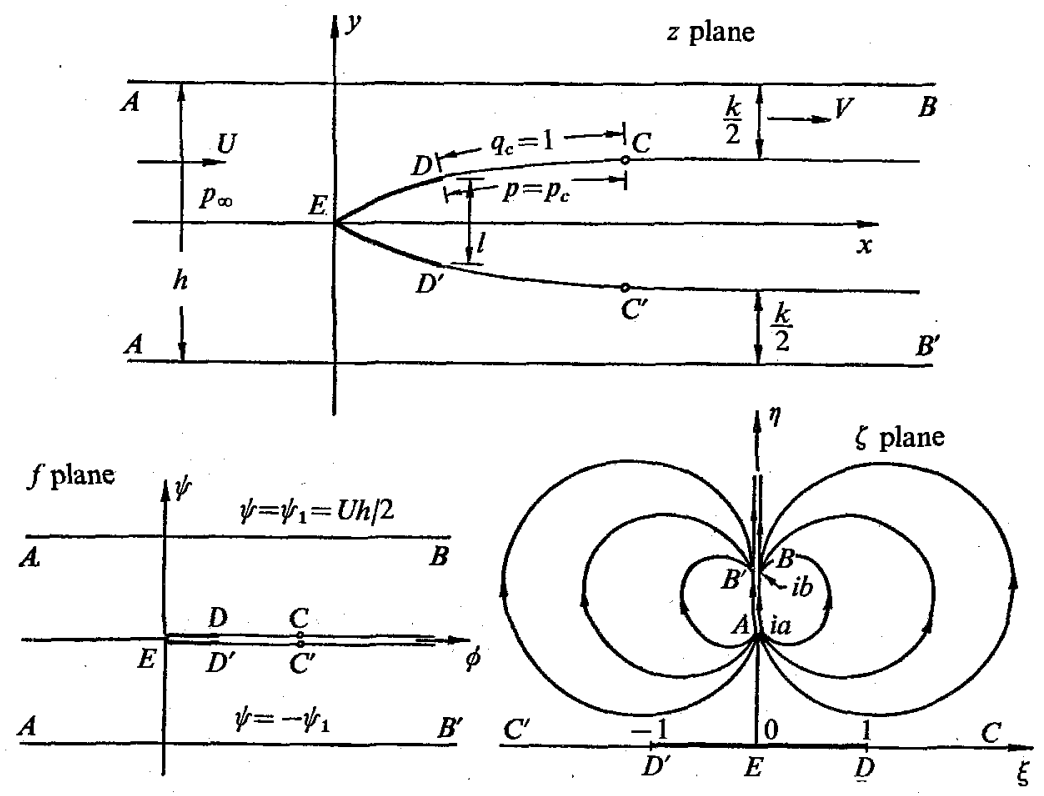

Figdre 2. The open-wake model for pure-drag flows in a tunnel.

plane $f=\phi+i \psi$ ( $\phi$ being the velocity potential and $\psi$ the stream function) is mapped into the upper half of the parametric plane $\zeta=\xi+i \eta$ by

$$
\frac{d f}{d \zeta}=\frac{A \zeta}{\left(\zeta^{2}+a^{2}\right)\left(\zeta^{2}+b^{2}\right)}, \quad A=\frac{1}{\pi} U h\left(b^{2}-a^{2}\right)
$$

in which the coefficient $A$ is determined by the jump of $\psi$ across the flow at the upstream or downstream infinity (point $A$ or $B$ ). The corresponding regions in the $z, f$, and $\zeta$ planes are shown in figure 2 .

Denote the $x, y$ velocity components by $u, v$, and the complex velocity by

$$
w=u-i v=\frac{d f}{d z}=q e^{-i \theta}, \quad q=|w|, \quad \theta=\tan ^{-1}(v / u)
$$


Then, if $s$ is the arc length measured from $E$ along $E D, \theta=\beta(s)$, say, is known on the body surface, and $q$ is known and equal to $q_{c}$ on the cavity boundary $D C$ and $D^{\prime} C^{\prime}$. For brevity, $q_{c}$ will be normalized henceforth to unity. In terms of the logarithmic hodograph variable

$$
\omega=\tau+i \theta=\log \mathrm{I} / w, \quad \tau=\log \mathrm{I} / q,
$$

the problem becomes the following Riemann-Hilbert boundary problem:

$$
\begin{gathered}
\theta(\xi, 0+)=\theta^{+}(\xi)=\beta(s(\xi)) \quad(|\xi|<1), \\
\tau(\xi, 0+)=0 \quad(|\xi|>1), \\
\omega=O(1 / \zeta) \quad \text { as } \quad|\zeta| \rightarrow \infty,
\end{gathered}
$$

in which we specify $s(-\xi)=-s(\xi)$, and $\beta(-s)=-\beta(s)$. We shall also designate $\beta(\xi)=\beta(s(\xi))$, with $\beta(-\xi)=-\beta(\xi)$. The solution of this problem is

$$
\omega(\zeta)=\frac{1}{i \pi}\left(\zeta^{2}-1\right)^{\frac{1}{2}} \int_{-1}^{1} \frac{\beta(\xi) d \xi}{(\xi-\zeta)\left(1-\xi^{2}\right)^{\frac{1}{2}}} \quad(\operatorname{Im} \zeta>0),
$$

in which the function $\left(\zeta^{2}-1\right)^{\frac{1}{2}}$ is analytic in the $\zeta$ plane cut along the $\xi$ axis from -1 to 1 , and tends to $\zeta$ as $|\zeta| \rightarrow \infty$. The last condition in (22) is also satisfied since the integral in (23) is $O\left(\zeta^{-2}\right)$ as $|\zeta| \rightarrow \infty$, by virtue of $\beta(\xi)$ being odd in $\xi$. Finally, the boundary conditions on $w$ at the upstream and downstream infinity require

$$
\begin{aligned}
\log \frac{1}{U}=\omega(i a)= & \frac{2}{\pi}\left(1+a^{2}\right)^{\frac{1}{2}} \int_{0}^{1} \frac{\beta(\xi) \xi d \xi}{\left(\xi^{2}+a^{2}\right)\left(1-\xi^{2}\right)^{\frac{1}{2}}}, \\
& \log \frac{1}{V}=\omega(i b) .
\end{aligned}
$$

Equations (19) and (23) provide a parametric solution $f=f(\zeta), \omega=\omega(\zeta)$. The physical plane is given by quadrature,

$$
z(\zeta)=\int_{0}^{\zeta} \frac{1}{w} \frac{d f}{d \zeta} d \zeta=\int_{0}^{\zeta} e^{\omega(\zeta)} \frac{d f}{d \zeta} d \zeta
$$

Since the base chord $D D^{\prime}$ is of length $l$, then $l=\operatorname{Im}[z(1)-z(-1)]$, or

$$
l=\operatorname{Im} \int_{-1}^{1} e^{\omega(\zeta)} \frac{d f}{d \zeta} d \zeta
$$

and hence, after substituting (19) in (27a),

$$
\lambda=\frac{l}{h}=\operatorname{Im} \frac{U}{\pi} \int_{-1}^{1} e^{\omega(\zeta)}\left[\frac{1}{\zeta^{2}+a^{2}}-\frac{1}{\zeta^{2}+b^{2}}\right] \zeta d \zeta .
$$

Now, on the body surface, as $\eta \rightarrow 0+$,

$$
\omega(\xi+i 0)=\Gamma(\xi)+i \beta(\xi), \quad \Gamma(\xi)=\frac{1}{\pi} \oint_{-1}^{1}\left(\frac{1-\xi^{2}}{1-t^{2}}\right)^{\frac{1}{2}} \frac{\beta(t) d t}{t-\xi},
$$

where $C$ over the integral sign indicates the Cauchy principal value. Thus the arc length $s$, measured from $E$ along $E D$, is

$$
s(\xi)=\int_{0}^{\xi} e^{\Gamma(\xi)} \frac{d f}{d \xi} d \xi \quad(0 \leqslant \xi \leqslant 1) .
$$


The drag coefficient is given by (9), or after setting $q_{c}=1$,

$$
C_{D}(\sigma, \lambda)=\frac{1}{\lambda}\left(\frac{V}{U}-1\right)\left(\frac{1}{U V}-1\right)
$$

The above solution may be regarded either as a direct (physical), or an inverse problem. The direct problem is prescribed by the set of quantities,

$$
P[\beta(s), \sigma, \lambda] \quad(0<s<S=s(1)),
$$

in which $\beta(s)$ is a known function of the arc length $s, \sigma$ is taken to be greater than the blockage constant $\sigma_{*}$ for fixed $\lambda(=l / h)<1$. The inverse problem is specified by

$$
P^{\prime}[\beta(\xi), a, b] \quad(-1<\xi<1),
$$

in which $\beta(\xi)$ is a given function of $\xi$ and $0<a<b$. The inverse problem is seen to be fully determined, since if the quantities $P^{\prime}$ are prescribed, then (24), (25) provide $U$ and $V,(23)$ determines $\omega(\zeta),(27)$ fixes $\lambda, z$ is given by (26), and finally the $C_{D}$ follows from (30). On the other hand, in the direct problem with fixed detachment (from a sharp corner of the obstacle), $s(\xi)$ and $\beta(\xi)=\beta(s(\xi)$ ) are not known a priori. Consequently its solution involves a non-linear integral equation (29) for $s(\xi)$ together with two parameters $a, b$, which must be evaluated under two functional conditions (24) and (27) for fixed $U$ and $l / h$. (Note that $U=(1+\sigma)^{-\frac{1}{2}}$.) In the case of smooth detachment (when the body curvature is finite on both sides of the detachment point, such as the detachment from a circular cylinder), an additional condition is required. The classical condition is that of Villat (1914), which can be written as $\left(\zeta^{2}-1\right)^{\frac{1}{2}} \omega^{\prime}(\zeta) \rightarrow 0$ as $\zeta \rightarrow 1$. It should be noted that $V$ cannot be arbitrary in problem $P$; instead it is fixed by (25) after $a, b$ and $\beta(\xi)$ are solved. Numerical methods for solution of the direct problem have been developed for the unbounded flow case by various authors (see e.g. Birkhoff \& Zarantonello 1957; Gilbarg 1961; Wu 1968). Furthermore; the approximate numerical scheme devised by Wu \& Wang (1964b) has been found to be very effective. These methods can also be applied to the present problem of wall effects.

Of particular interest is the simple case of symmetric wedges since in this case $\beta$ is constant and the parameters become uncoupled ( $U$ is then a function of $a$ only, see (24)). Consequently, the solution is greatly simplified by considering a mixed type problem $P^{\prime \prime}[\beta, \sigma, b]$, the direct problem being solved by simple cross-plotting. We proceed to present the details as follows.

For a symmetric wedge of half vertex angle $\beta \pi$, we have

$$
\beta(\xi)=\text { constant }=\beta \pi \quad(0<\xi<1) .
$$

Then (23) can be readily integrated, giving

$$
w(\zeta)=e^{-\omega}=e^{-i \beta \pi}\left\{\zeta /\left[1+\left(1-\zeta^{2}\right)^{\frac{1}{2}}\right]\right\}^{2 \beta} .
$$

Hence, conditions (24) and (25) become

$$
\begin{gathered}
U=U(a)=\left\{a /\left[1+\left(1+a^{2}\right)^{\frac{1}{2}}\right]\right\}^{2 \beta}, \quad \text { or } \quad a=a(U) \equiv 2\left(U^{-1 / 2 \beta}-U^{1 / 2 \beta}\right)^{-1} \\
V=U(b), \quad \text { or } \quad b=a(V) .
\end{gathered}
$$


Furthermore, (27) gives the base-chord to channel-width ratio as

$$
\dot{\lambda}=l / h=U[F(U)-F(V)]
$$

where $\quad F(U)=F(U(a)) \equiv \frac{2}{\pi} \sin \beta \pi \int_{0}^{1}\left[1+\left(1-\zeta^{2}\right)^{\frac{1}{2}}\right]^{2 \beta} \frac{\zeta^{1-2 \beta}}{\zeta^{2}+a^{2}} d \zeta$

and $F(V)=F(U(b))$, i.e. with $a$ in $(37 b)$ replaced by $b$.

For the direct problem $P[\beta, \sigma, \lambda], a$ can first be computed from (35) noting that $U=(1+\sigma)^{-\frac{1}{2}}$. Then $b$ can be determined from $(37 a, b)$, and finally $V$ is given by $(36)$, and $C_{D}$ by (30). The integral (37b) can only be integrated in closed form

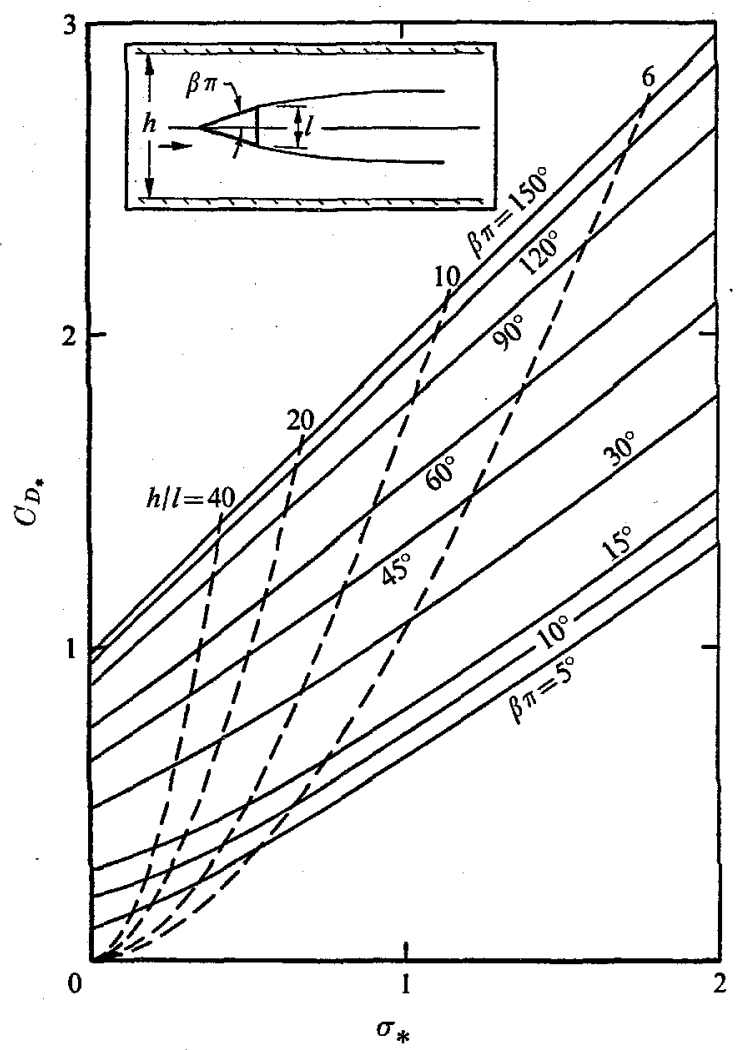

Figure 3. Choked-flow drag $C_{D_{*}}(\beta, l / h)$ of wedges $v s$. the choked cavitation number $\sigma_{*}(\beta, l / h)$. Cavity is finite in length for $\sigma>\sigma_{*}$.

when $\beta=m / n$ ( $m$ and $n$ being integers), since then appropriate changes of variables can reduce the integrand to a rational fraction. In particular, for the flat plate, $\beta=\frac{1}{2}$, the result is rather simple:

$$
\lambda=\frac{l}{h}=\left(1-\frac{U}{V}\right)+\frac{2 U}{\pi}\left\{\left(\frac{1}{V}-V\right) \tan ^{-1} V-\left(\frac{1}{U}-U\right) \tan ^{-1} U\right\} .
$$

However, for a wide range of $\beta$, it is more convenient to evaluate the integral numerically. 
The choked flow state is reached as $b \rightarrow \infty$, or equivalently, as $V \rightarrow 1$. The corresponding limit of $a$ and $U$, for fixed $\beta$ and $\lambda$, will be denoted by $a_{*}$ and $U_{*}$ which are related by $U_{*}=U\left(a_{*}\right), U(a)$ being given by (35). By letting $b \rightarrow \infty$ in (37), we have $F(V) \rightarrow 0$, and hence

$$
\lambda=U_{*} F\left(U\left(a_{*}\right)\right)
$$

which determines $a_{*}=a_{*}(\beta, \lambda)$. The corresponding drag coefficient at the choked condition is, by (30),

$$
C_{D *}=\frac{h}{l}\left(\frac{1}{U_{*}}-1\right)^{2}=\frac{1}{\lambda}\left[\left(1+\sigma_{*}\right)^{\frac{1}{2}}-1\right]^{2} .
$$

The choked flow results (39) and (40) have been computed numerically for several values of $\beta$, as shown in figure 3 . The result shows that, for $0<\beta<1, \lambda$ and $C_{D_{*}}$

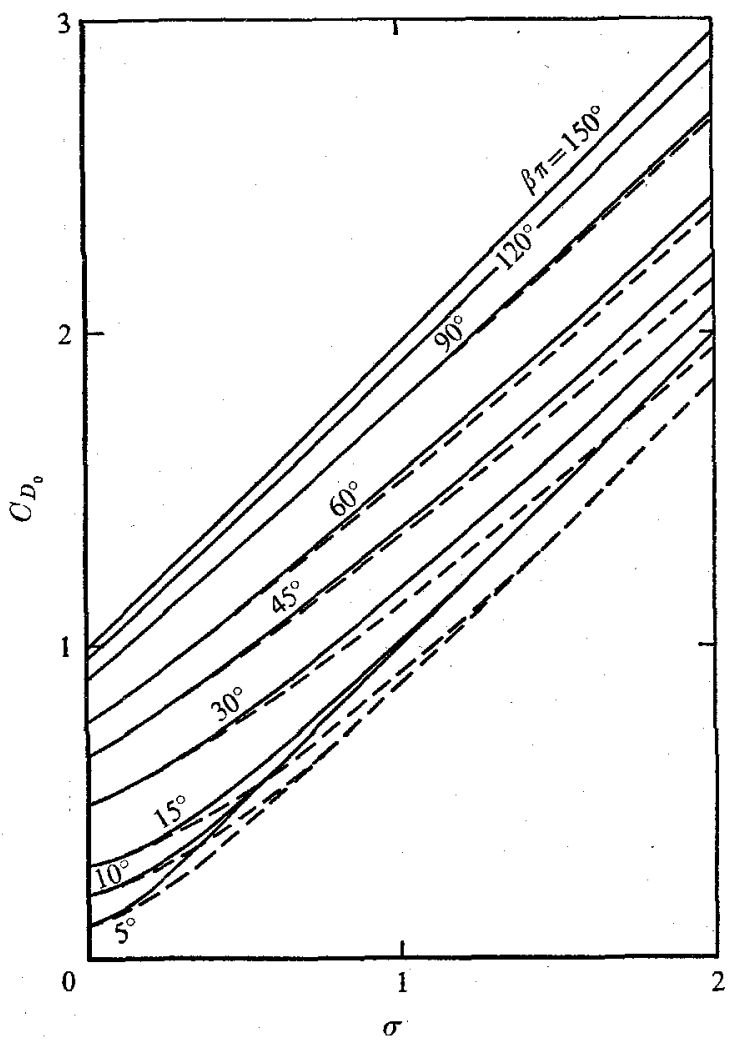

FigURE 4. Drag coefficients of wedges in unbounded flow $(h / l=\infty)$ based on different theoretical models: _ _, open-wake model; ... ., Riabouchinsky model.

both increase monotonically with decreasing $a_{*}$ (or with increasing choking cavitation number $\sigma_{*}$ ). With this behaviour of $\lambda$ it also follows from (37), by simple comparison, that before the tunnel is choked $a$ and $U$ are such that $a<a_{*}, U<U_{*}$ (and hence $\sigma>\sigma_{*}$ ).

The wall effect diminishes as $\lambda \rightarrow 0$; the limit is reached as $b \rightarrow a$ (or $V \rightarrow U$ ). In this limit, the drag coefficient $C_{D}(\sigma, \beta, \lambda)$ tends to its value in unbounded 
flow $C_{D_{0}}(\sigma, \beta)$, which can be deduced from (30), (36) and (37) by applying l'Hospital's rule, giving

$$
C_{D_{0}}(\sigma, \beta)=\frac{1}{U^{2}}\left(\frac{1}{U}-U\right) /\left(\frac{\partial \lambda}{\partial b}\right)_{b=a}\left(\frac{\partial b}{\partial V}\right)_{V=U}=\left(\frac{U^{-1}-U}{U^{-1 / \beta}-U^{1 / \beta}}\right) \frac{4 \beta(1+\sigma)}{a^{4} C_{0}}
$$

where $C_{0}=-d F(U(a)) / d a^{2}, F(U)$ being given by $(37 b)$. This result has been obtained previously by $\mathrm{Wu} \& \mathrm{Wang}(1964 a)$. The above drag coefficient $C_{D_{0}}(\sigma, \beta)$ for unbounded flow is shown in figure 4.

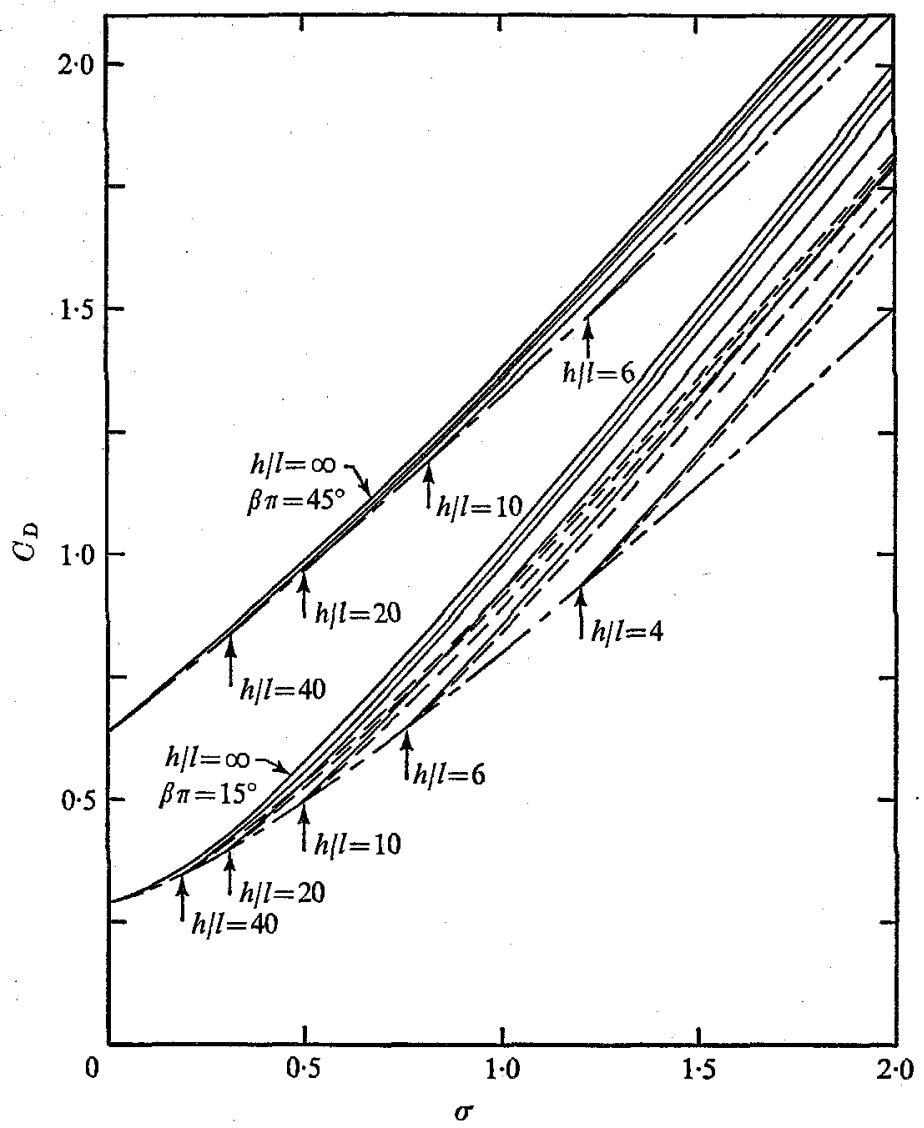

Figure 5. Comparisons of the drag coefficient for $30^{\circ}$ and $90^{\circ}$ wedges based on the different models: - - open-wake model; _- - , choked flow; - ...., Riabouchinsky model.

The drag coefficient $C_{D}(\sigma, \beta, \lambda)$ has been calculated from (30), (35)-(37) for $\sigma<\sigma_{*}$ and various $\beta$ and $\lambda$. The numerical computation was carried out with an IBM-360 machine, using the straightforward iteration scheme described earlier for the direct problem. With certain transformations of the integration variables administered for small values of $\beta$ and $\sigma$, convergence of the iterations was found satisfactory for all values of $\sigma, \beta$, and $\lambda$, the errors allowed being less than $10^{-6}$. The final numerical results are shown in figure 5 for two representative examples $\beta \pi=15^{\circ}$ and $45^{\circ}$. For a complete coverage of the results reference 
is made to $\mathrm{Wu}$, Whitney \& $\operatorname{Lin}(1969)$. Several salient features of the wall effect in cavity flows may be noted from these results. First, the effect of the presence of the tunnel walls is to make $C_{D}$ lower than that in an unbounded flow at the same cavitation number. The physical reason for this result has been given earlier.

Another remarkable feature of the results is that the percentage drag reduction due to the wall, for fixed $\sigma$ and $\lambda$, actually increases with decreasing wedge angle. For the half vertex angle $\beta \pi>90^{\circ}$, the wall effect becomes exceedingly small. This would imply that wall effects are in general more significant for thinner bodies in cavity flows, other conditions being equal. Such a general conclusion may seem to be surprising at a first glance. However, it is physically reasonable that the pressure reduction over the wetted surface of a thin body may be felt over a longer stretch than for blunt bodies. This is borne out by the experimental measurements to be made explicit later. Another possible reason is that the curvature singularity of the cavity boundary at separation becomes weaker as the body thickness ratio decreases, thus causing a greater pressure reduction on the wetted surface of the body.

A further point of interest is that the drag reduction (absolute difference) is very insensitive to $\sigma\left(>\sigma_{*}\right)$ for given $\beta$ and $\lambda$. This finding is of practical value, since, by this rule, the problem of estimating the drag reduction involved in $C_{D}(\sigma, \beta, \lambda)$ for $\sigma>\sigma_{*}(\beta, \lambda)$ (with a finite cavity) is reduced simply to a comparison between the choked flow drag coefficient $C_{D_{*}}\left(\sigma_{*}, \beta, \lambda\right)$ and the unbounded flow value $C_{D}(\sigma, \beta, 0)$ at $\sigma=\sigma_{*}$. More specifically, this rule means that, for $\sigma \geqslant \sigma_{*}$,

$$
C_{D}(\sigma, \beta, 0)-C_{D}(\sigma, \beta, \lambda)=C_{D}\left(\sigma_{*}, \beta, 0\right)-C_{D *}\left(\sigma_{*}, \beta, \lambda\right)
$$

For experimental purposes, however, this rule is insufficient, since $C_{D}(\sigma, \beta, 0)$ for unbounded flow is often not known. For this reason, other methods for correcting the wall effects will be explored in $\$ 6$. In order to ascertain the accuracy of the open-wake model, the above results will be further examined by comparison with the Riabouchinsky model and the experiments.

\section{Riabouchinsky model}

We next apply the Riabouchinsky model to evaluate the pure-drag cavity flow past a symmetrical body of arbitrary shape placed in a tunnel. The particular case of the flat plate has been dealt with by Birkhoff et al. (1952).

The corresponding regions in the $z$ and $f$ planes are shown together with the parametric $\zeta$ plane in figure 6 . The upper half strip in the $f$ plane is mapped into the upper half $\zeta$ plane by the general Schwarz-Christoffel transformation (see Gilbarg 1949):

$$
\frac{d f}{d \zeta}=\frac{A \zeta}{\left(\zeta^{2}+a^{2}\right)\left(\zeta^{2}+b^{2}\right)^{\frac{1}{2}}}, \quad A=\frac{1}{\pi} U h\left(b^{2}-a^{2}\right)^{\frac{1}{2}}
$$

in which the coefficient $A$ is determined by the local behaviour of $f$ at the point $\zeta=i a$. The function $\left(\zeta^{2}+b^{2}\right)^{\frac{1}{2}}$ is analytic in the $\zeta$ plane cut from $\zeta=-i b$ to $\zeta=i b$, and $\left(\zeta^{2}+b^{2}\right)^{\frac{1}{2}} \rightarrow \zeta$ as $|\zeta| \rightarrow \infty$. The boundary values of $\omega=\tau+i \theta$ again 
assume the same form as (22), though the symbol $\zeta=\xi+i \eta$ must be referred to the present case. (Here we note that $\theta=0$ on $B C$ due to the flow symmetry.) It therefore follows that the parametrie solution $\omega=\omega(\zeta)$, the velocity condition $\omega(i a)=-\log U, z=z(\zeta)$, the base chord $l$, the arc length $s(\xi)$ can again be expressed formally by (23), (24), (26), (27a), and (29), respectively. The velocity $V$ now gives the maximum flow velocity along the wall at point $B$. Thus formally the numerical solution for an arbitrary body shape can be carried out by the same procedure as described in the previous case. This completes our solution.

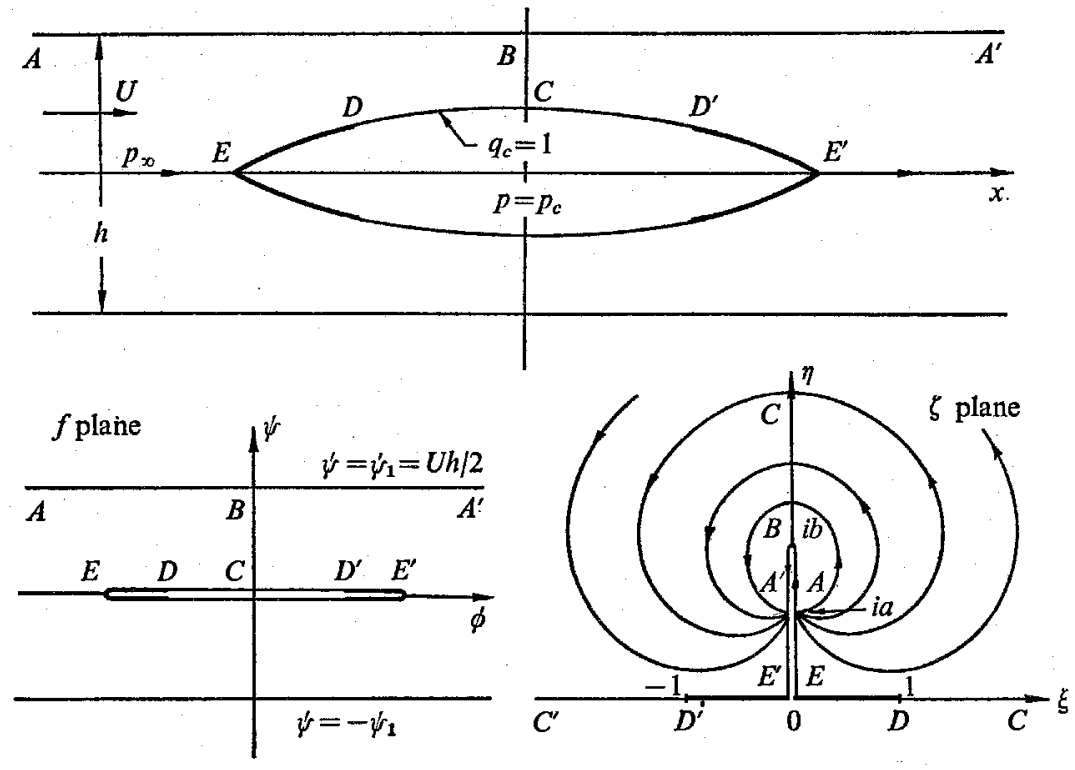

FIGURE 6. The Riabouchinsky model for pure-drag cavity flows in a tunnel.

The drag on the body is given by

$D=2 \operatorname{Im} \int_{Z_{E}}^{Z_{D}}\left(p-p_{c}\right) d z=\operatorname{Im} \rho \int_{0}^{1} \frac{1-w \bar{w}}{w} \frac{d f}{d \zeta} d \zeta=\operatorname{Im} \rho \int_{0}^{1}\left(\frac{1}{w}+w\right) \frac{d f}{d \zeta} d \zeta$,

in which use has been made of the property $\operatorname{Im}(w+\bar{w})=0$ for $\zeta$ real.

For a symmetric wedge of half vertex angle $\beta \pi, w(\zeta)$ is again given by (34), and (35) remains valid to assure $w(i a)=U$. The ratio $\lambda=l / h$; by $(27 a)$, now becomes

$$
\lambda=\frac{2 U}{\pi}(\sin \beta \pi)\left(b^{2}-a^{2}\right)^{\frac{1}{2}} \int_{0}^{1} \frac{\left(1+\left(1-\zeta^{2}\right)^{\frac{1}{2}}\right)^{2 \beta} \zeta^{1-2 \beta}}{\left(\zeta^{2}+a^{2}\right)\left(\zeta^{2}+b^{2}\right)^{\frac{1}{2}}} d \zeta .
$$

Finally, (44) gives the drag coefficient, upon eliminating $\lambda$, as

$$
\begin{gathered}
C_{D}=(1+\sigma)\left[1-I_{-}(a, b) / I_{+}(a, b)\right], \\
I_{ \pm}(a, b)=\int_{0}^{1} \frac{\left[1 \pm\left(1-\zeta^{2}\right)^{\frac{1}{2}}\right]^{2 \beta} \zeta^{1-2 \beta}}{\left(\zeta^{2}+a^{2}\right)\left(\zeta^{2}+b^{2}\right)^{\frac{1}{2}}} d \zeta .
\end{gathered}
$$

The numerical method of solution for arbitrary angle $\beta$ is again very much the same as described in the previous case.

The choked flow state is reached as $b \rightarrow \infty$. The limit of $\lambda$ as $b \rightarrow \infty$ is obviously 
identical to (39). Furthermore, we derive from (46) and (45) the corresponding limit of $C_{D}$ as

$$
C_{D_{*}}=\frac{2 \sin \beta \pi}{\pi U_{*} \lambda} \int_{0}^{1}\left[\left(\frac{1+\left(1-\zeta^{2}\right)^{\frac{1}{2}}}{\zeta}\right)^{2 \beta}-\left(\frac{1-\left(1-\zeta^{2}\right)^{\frac{1}{2}}}{\zeta}\right)^{2 \beta}\right] \frac{\zeta d \zeta}{\zeta^{2}+a_{*}^{2}}=\frac{1}{\lambda}\left(\frac{1}{U_{*}}-1\right)^{2}
$$

upon integration with appropriate change of variables $\left(t=\left[1-\left(1-\zeta^{2}\right)^{\frac{1}{2}}\right] / \zeta\right.$, and with use of the theorem of residues in the complex $t$ plane). This result agrees with the momentum theorem (10), as should be expected.

At the other extreme, the unbounded flow limit is readily deduced from (46), $b \rightarrow a$, with the corresponding drag coefficient given by

$$
C_{D_{0}}(\beta, \sigma)=(1+\sigma)\left[I-I_{-}(a, a) / I_{+}(a, a)\right] .
$$

This result, as plotted in figure 4 together with the open-wake model, shows that the theoretical $C_{D_{0}}$ in unbounded flow, based on the Riabouchinsky model, is smaller than that predicted by the open-wake model, and that this difference becomes increasingly more appreciable with decreasing wedge angle.

The drag coefficient $C_{D}(\sigma, \beta, \lambda)$ in bounded flows has been calculated from (45), (46) over the same range of the parameters as in the preceding case of the openwake model, employing similar numerical methods. As can be seen in the two examples shown in figure 5, the important features of the wall effect described in $\$ 3$ are very much common to both of the two theoretical flow models. However, since the difference between their predicted values of $C_{D}$ is especially marked for small $\lambda$ at moderate to high $\sigma$ (away from $\sigma_{*}$ ), discrimination between these models must necessarily come from further precise experimental studies. Such an attempt will be discussed in $\S \S 7$ and 8 .

\section{Re-entrant jet model}

The re-entrant jet model has been adopted by Gurevich (1953) to evaluate symmetric cavity flows past a wedge placed in a straight channel. In what follows, the general case of a symmetric body of arbitrary shape is treated by using this model.

The corresponding regions in the $z$ and $f$ planes are shown in figure 7. Though a parametric plane similar to those of the previous two models (i.e. with the body surface and cavity boundaries spanning the entire real axis of the parametric plane) can also be constructed, the present $\zeta=\xi+i \eta$ plane has certain simplifications. The upper half strip of the $f$ plane is mapped into the second quadrant of the $\zeta$ plane by the transformation,

$$
\frac{d f}{d \zeta}=\frac{A \zeta\left(\zeta^{2}-c^{2}\right)}{\left(\zeta^{2}-a^{2}\right)\left(\zeta^{2}-b^{2}\right)}
$$

where $A$ is a positive real constant. By this formula $f$ can be continued analy tically into the entire $\zeta$ plane (by virtue of $\operatorname{Im} f=0$ on $\xi=0$ ). From the singular behaviour of $f$ at $\zeta=a, b$, and $\infty$, it follows that

$$
\begin{aligned}
U h & =\pi A\left(c^{2}-a^{2}\right) /\left(b^{2}-a^{2}\right), \\
V h & =\pi A\left(c^{2}-b^{2}\right) /\left(b^{2}-a^{2}\right), \\
l_{j} & =\pi A .
\end{aligned}
$$


Condition (51) assures that the flow in the downstream tunnel is simply covered. From (50) and (51) it also follows that

$$
V / U=\left(c^{2}-b^{2}\right) /\left(c^{2}-a^{2}\right) .
$$

From (50)-(52) follows also the continuity condition $(U-V) h=l_{j}$.
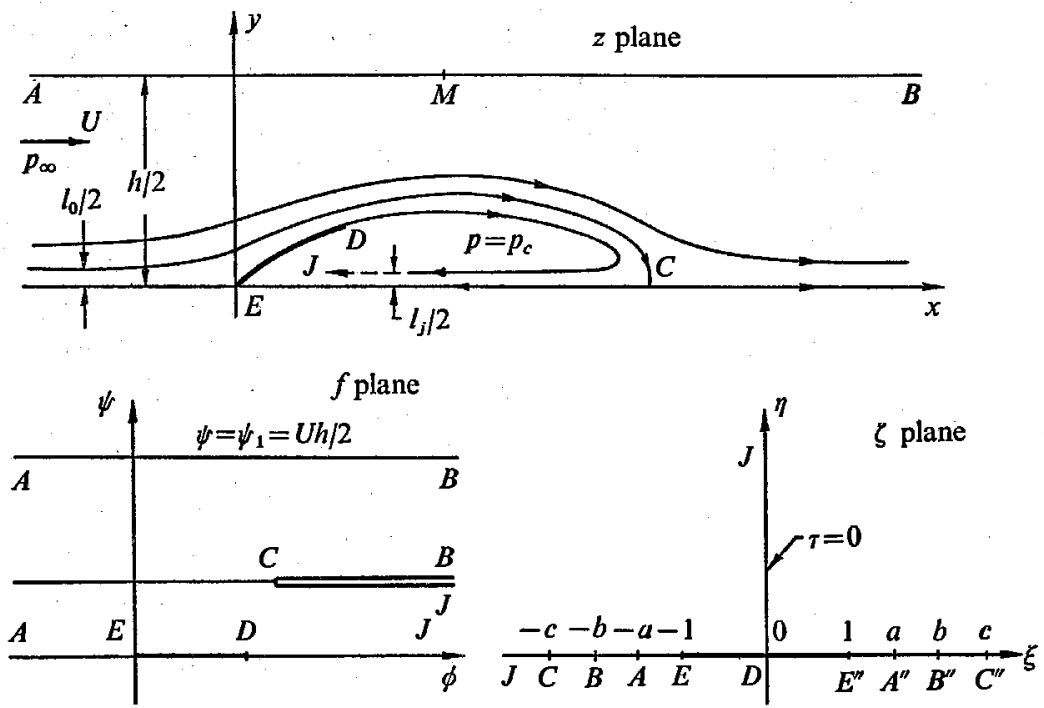

Figure 7. The re-entrant jet model for pure-drag cavity flows in a tunnel.

The boundary conditions of $\omega=\tau+i \theta$ are

$$
\left.\begin{array}{rlrl}
\theta^{+}(\xi)=\theta(\xi,+0) & =-\pi & & (\xi<-c), \\
& =0 & & (-c<\xi<-1), \\
& =\beta(\xi) & & (-1<\xi<0), \\
\tau(0, \eta) & =0 & & (\eta>0) .
\end{array}\right\}
$$

The last condition of (54) enables $\omega(\zeta)$ to be analytically continued into the first quadrant of the $\zeta$ plane by $\omega(-\bar{\zeta})=-\overline{\omega(\zeta)} \cdot(\omega(\zeta)$ can further be continued into the lower half $\zeta$ plane by $\omega(\bar{\zeta})=\overline{\omega(\zeta)}$, so that $\theta$ is odd in $\eta$. The lower half flow field then corresponds to the fourth quadrant of the $\zeta$ plane.) After this continuation, $\theta$ is prescribed as an even function of $\xi$, for the entire $\xi$ axis. The solution $\omega(\zeta)$ is then given by the Poisson integral,

$$
\omega(\zeta)=\frac{1}{\pi} \int_{-\infty}^{\infty} \frac{\theta^{+}(\xi) d \xi}{\xi-\zeta}=\log \left(\frac{c-\zeta}{c+\zeta}\right)+\frac{1}{\pi} \int_{-1}^{1} \frac{\beta(\xi) d \xi}{\xi-\zeta} .
$$

Hence,

$$
w(\zeta)=e^{-\omega}=\left(\frac{c+\zeta}{c-\zeta}\right) e^{-\Omega(\zeta)}, \quad \Omega(\zeta)=\frac{1}{\pi} \int_{-1}^{1} \frac{\beta(\xi) d \xi}{\xi-\zeta} .
$$

The boundary conditions of $\omega$ at points $A$ and $B$ require that

$$
U=\left(\frac{c-a}{c+a}\right) e^{-\Omega(-a)}, \quad V=\left(\frac{c-b}{c+b}\right) e^{-\Omega(-b)} .
$$


Upon substituting the above $U, V$ into (53), there results

$$
(c+b) /(c+a)=\exp \left\{\frac{1}{2}[\Omega(-a)-\Omega(-b)]\right\},
$$

from which $c$ can be determined as a function of $a, b$; or $c=c(a, b)$ say.

The physical plane is given by

$$
z(\zeta)=\int_{-1}^{\zeta} \frac{1}{w} \frac{d f}{d \zeta} d \zeta=A \int_{-1}^{\zeta} e^{\Omega(\zeta)} \nu(\zeta ; a, b) d \zeta, \quad \nu=\frac{-\zeta(\zeta-c)^{2}}{\left(\zeta^{2}-a^{2}\right)\left(\zeta^{2}-b^{2}\right)} .
$$

The half-base chord is $l / 2=\operatorname{Im} z(0)$; hence, upon using (50),

$$
\lambda=\frac{l}{h}=\frac{2 U}{\pi}\left(\frac{b^{2}-a^{2}}{c^{2}-a^{2}}\right) \operatorname{Im} \int_{-1}^{0} e^{\Omega(\zeta)} \nu(\zeta ; a, b) d \zeta .
$$

The arc length $s$ measured from $E$ along $E D$ is

$$
s(\xi)=A \int_{-1}^{\xi} e^{\Gamma(\xi)} \nu(\xi ; a, b) d \xi, \quad \Gamma(\xi)=\frac{1}{\pi} \oint_{-1}^{1} \frac{\beta(t) d t}{t-\xi} .
$$

For the inverse problem with prescribed $P^{\prime}[\beta(\xi), a, b], c$ is determined by (58), $U$ by (57), $\lambda$ by $(60)$ and $s(\xi)$ by (61). Solution of the direct problem $P[\beta(s), \sigma, \lambda]$ can also proceed along the same lines as described for the previous two models; it is, however, more complicated here since this solution contains an extra parameter.

The drag coefficient has been given for general asymmetric flows by the momentum theorem (17). For the present problem, $q_{c}=1, \gamma=0$, and

$$
C_{D}=\frac{1}{\lambda}\left(1-\frac{V}{U}\right)\left(1+\frac{V+2}{U}\right)
$$

For a symmetric wedge of half vertex angle $\beta \pi, \Omega$ can be integrated to yield

$$
\Omega(\zeta)=\beta \log \left(\frac{\zeta-1}{\zeta+1}\right)
$$

which is defined in the $\zeta$ plane cut along the $\xi$ axis from $\zeta=-1$ to 1 , so that $\Omega \rightarrow-2 \beta \mid \zeta$ as $|\zeta| \rightarrow \infty$. Hence, by (57),

$$
U=\left(\frac{c-a}{c+a}\right)\left(\frac{a-1}{a+1}\right)^{\beta}, \quad V=\left(\frac{c-b}{c+b}\right)\left(\frac{b-1}{b+1}\right)^{\beta}
$$

and (58) becomes

$$
c=\frac{\kappa b-a}{1-\kappa}, \quad \kappa=\left(\frac{a-1}{a+1}\right)^{\beta / 2}\left(\frac{b+1}{b-1}\right)^{\beta / 2} \text {. }
$$

Upon substituting (63) in (60),

$$
\begin{aligned}
\lambda & =U[J(a, c)-J(b, c)] /\left(c^{2}-a^{2}\right), \\
J(a, c) & =\frac{2}{\pi} \sin \beta \pi \int_{0}^{1}\left(\frac{1+\zeta}{1-\zeta}\right)^{\beta} \frac{\zeta(\zeta+c)^{2}}{a^{2}-\zeta^{2}} d \zeta .
\end{aligned}
$$

Equations (64)-(66) determine $U, \lambda$ in terms of $a, b$, and vice versa.

The choked flow state is approached as $c \rightarrow \infty$, and $b \rightarrow \infty$. When both $b$ and $c$ are large compared with $a$, we deduce from (65) the relation,

$$
\frac{c}{b}=\frac{\kappa}{1-\kappa}\left[1+O\left(\frac{a}{b}\right)\right] \text { with } \kappa \simeq\left(\frac{a_{*}-1}{a_{*}+1}\right)^{\beta / 2} .
$$


Using (67) in (64), we obtain for $b \gg a$,

$$
U_{*}=\kappa^{2}, \quad V_{*}=2 \kappa-1 .
$$

The corresponding limit of $\lambda=l / h$ is simply

$$
\lambda=\frac{2 U_{*}}{\pi} \sin \beta \pi \int_{0}^{1}\left(\frac{1+\zeta}{1-\zeta}\right)^{\beta} \frac{\zeta d \zeta}{a_{*}^{2}-\zeta^{2}} .
$$

By substituting (68) in (62), we find

$$
C_{D_{*}}=\frac{1}{\lambda}\left(\frac{1}{U_{*}}-1\right)^{2},
$$

which is in agreement with the previous two flow models. From the requirement $U_{*}<1$ and $V_{*}>0$ it follows that $\kappa$ must lie in the range $\frac{1}{2}<\kappa<1$, and hence $a_{*}>(1+\gamma) /(1-\gamma), \gamma=2^{-2 / \beta}$.

The unbounded flow limit can be derived by letting $b \rightarrow a$, and by applying l'Hospital's rule to (65), giving

$$
c=\frac{1}{\beta}\left(a^{2}-1\right)-a
$$

hence, by (64),

$$
U=\frac{a^{2}-1-2 a \beta}{(a-1)^{1-\beta}(a+1)^{1+\beta}} .
$$

We further obtain, by first substituting (53) and (66) in (62), and then letting $b \rightarrow a$, for the drag coefficient in unbounded flow,

$$
C_{D_{0}}=2(1+U) /\left(U^{2} K\right)
$$

where

$$
K(a, c)=-\partial J(a, c) / \partial a^{2}
$$

and $J(a, c)$ is given by $(66 b)$.

\section{Wall correction formulae}

Since the ratio $\lambda=l / h$ is usually small in experimental practice, the asymptotic representation, for $\lambda$ small, of the preceding exact solutions can serve useful purposes for evaluating the wall effects and their corrections. The analysis of the asymptotic expansion is less complicated for symmetric wedges and will be carried out for two different flow models.

\subsection{Open-wake model}

For fixed $\sigma$ (hence $U$ ), the unbounded flow limit $(\lambda=0)$ of the drag coefficient $C_{D}(\sigma, \lambda)$ of wedges is obtained by letting $V \rightarrow U$ in (30) and (37a), giving, upon using l'Hospital's rule,

$$
C_{D}(\sigma, 0)=-U^{-2}\left(U^{-2}-1\right) /[d F(U) / d U] .
$$

If this equation is solved for $F^{\prime}(U)$, and integrated from $U$ to $V$, an alternative expression for $\lambda$ is obtained (using again (37a)):

$$
\lambda=U \int_{U}^{\nabla} \frac{u^{-2}\left(u^{-2}-1\right)}{C_{D}(\sigma(u), 0)} d u
$$


where $\sigma(u)=u^{-2}-1$. For a given body shape (the wedge angle), (75) determines $V$ implicitly as a function of $\sigma$ and $\lambda$.

We next seek a partial differential equation for $C_{D}(\sigma, \lambda)$. Partial differentiation of (75) and $C_{D}(\sigma, \lambda)$ given by (30) with respect to $\sigma$ and $\lambda$ and elimination of terms involving $V$ gives

$$
\begin{aligned}
\frac{2(1+\sigma)}{\sigma} C_{D}(\sigma, 0) \frac{\partial}{\partial \sigma} & \left(C_{D}(\sigma, \lambda)\right)+\left[1-\frac{\lambda}{\sigma} C_{D}(\sigma, 0)\right] \frac{\partial}{\partial \lambda}\left(C_{D}(\sigma, \lambda)\right) \\
& =\frac{1}{\lambda}\left[C_{D}(\sigma, 0)-C_{D}(\sigma, \lambda)\right]+\frac{2}{\sigma} C_{D}(\sigma, 0) C_{D}(\sigma, \lambda) .
\end{aligned}
$$

In the limit as $\lambda \rightarrow 0$, this equation reduces to

$$
\frac{\partial}{\partial \lambda}\left(C_{D}(\sigma, 0)\right)+\left(\frac{1+\sigma}{\sigma}\right) C_{D}(\sigma, 0) \frac{\partial}{\partial \sigma}\left(C_{D}(\sigma, 0)\right)=\frac{1}{\sigma} C_{D}^{2}(\sigma, 0) .
$$

For fixed $\sigma$, (76) gives an estimate of the dependence of $C_{D}$ on $\lambda$, namely $\partial C_{D} / \partial \lambda$, provided both $C_{D}$ and $\partial C_{D} / \partial \sigma$ are known. These quantities may be found either from theory or experiments (assuming $C_{D}(\sigma, 0)=C_{D}(\sigma, \lambda)+O(\lambda)$ ), although the calculation of $\partial C_{D} / \partial \sigma$ would require estimating a derivative from experimental data, which can be rather inaccurate.

A more useful result follows by integrating (76) from $\sigma^{\prime}$ to $\sigma>\sigma^{\prime}\left(\sigma-\sigma^{\prime}=O(\lambda)\right)$ along the mathematical characteristies of (76),

$$
\frac{d \sigma}{d \lambda}=\left(\frac{1+\sigma}{\sigma}\right) C_{D}(\sigma, 0), \quad \frac{d C_{D}}{d \sigma}=\frac{C_{D}(\sigma, 0)}{1+\sigma}
$$

giving

$$
C_{D}\left(\sigma^{\prime}, 0\right)=\left(\frac{1+\sigma^{\prime}}{1+\sigma}\right) C_{D}(\sigma, \lambda)+O\left(\lambda^{2}\right)
$$

where

$$
\sigma^{\prime}=\sigma-\left(\frac{1+\sigma^{\prime}}{\sigma^{\prime}}\right) C_{D}\left(\sigma^{\prime}, 0\right) \lambda+O\left(\lambda^{2}\right)
$$

or, to the same order of accuracy,

$$
\sigma^{\prime}=\sigma-\left(\frac{1+\sigma}{\sigma}\right) C_{D}(\sigma, \lambda) \lambda+O\left(\lambda^{2}\right)
$$

This two-way correction rule (first derived by Whitney 1969) takes a measured drag coefficient $C_{D}(\sigma, \lambda)$, in a tunnel of known $\lambda$, and converts it by (77) and (78b) to an estimated unbounded drag coefficient $C_{D}\left(\sigma^{\prime}, 0\right)$ in unbounded flow $(\lambda=0)$ at a different cavitation number, $\sigma^{\prime}$, given by $(78 b)$. The process may also be reversed by use of (77), (78a) instead of (77), (78b).

An example of the use of this rule in estimating unbounded drag coefficients from theoretically calculated data, $C_{D}(\sigma, \lambda)$, is shown in figure 8 for $\beta \pi=15^{\circ}$. The agreement of predicted estimates with calculated values of $C_{D}\left(\sigma^{\prime}, 0\right)$ is found to be excellent for all angles, with $\lambda$ up to $\frac{1}{6}$ and $\sigma$ up to 1 .

As was pointed out earlier, estimates of $C_{D}(\sigma, \lambda)$ can be readily obtained if good approximations to $C_{D}(\sigma, 0)$ are known. For example, for wedges with $\beta \pi>60^{\circ}$, a good approximation of $C_{D}(\sigma, 0)$ for $\sigma<1$ is

$$
C_{D}(\sigma, 0)=C_{0}(\beta)(1+\sigma) \text {. }
$$


Then (75) can be integrated to yield $\lambda=\left(U / C_{0}\right)\left(U^{-1}+U-V^{-1}-V\right)$, and hence, by $(30)$,

$$
C_{D}(\sigma, \lambda)=C_{0} U^{-2}=C_{0}(1+\sigma)=C_{D}(\sigma, 0) .
$$

Thus, there is no correction for wall effect if $C_{D}(\sigma, 0)$ obeys the linear relationship (79a) exactly, and it is reasonable to expect that the correction is small if (79a) is satisfied approximately. This is confirmed by numerical calculations. In this case, the correction rule (77), (78) simply shifts $C_{D}(\sigma, \lambda)$ along the same curve of $C_{D}(o, 0)$ from one value $\sigma$ to another value $\sigma^{\prime}$.

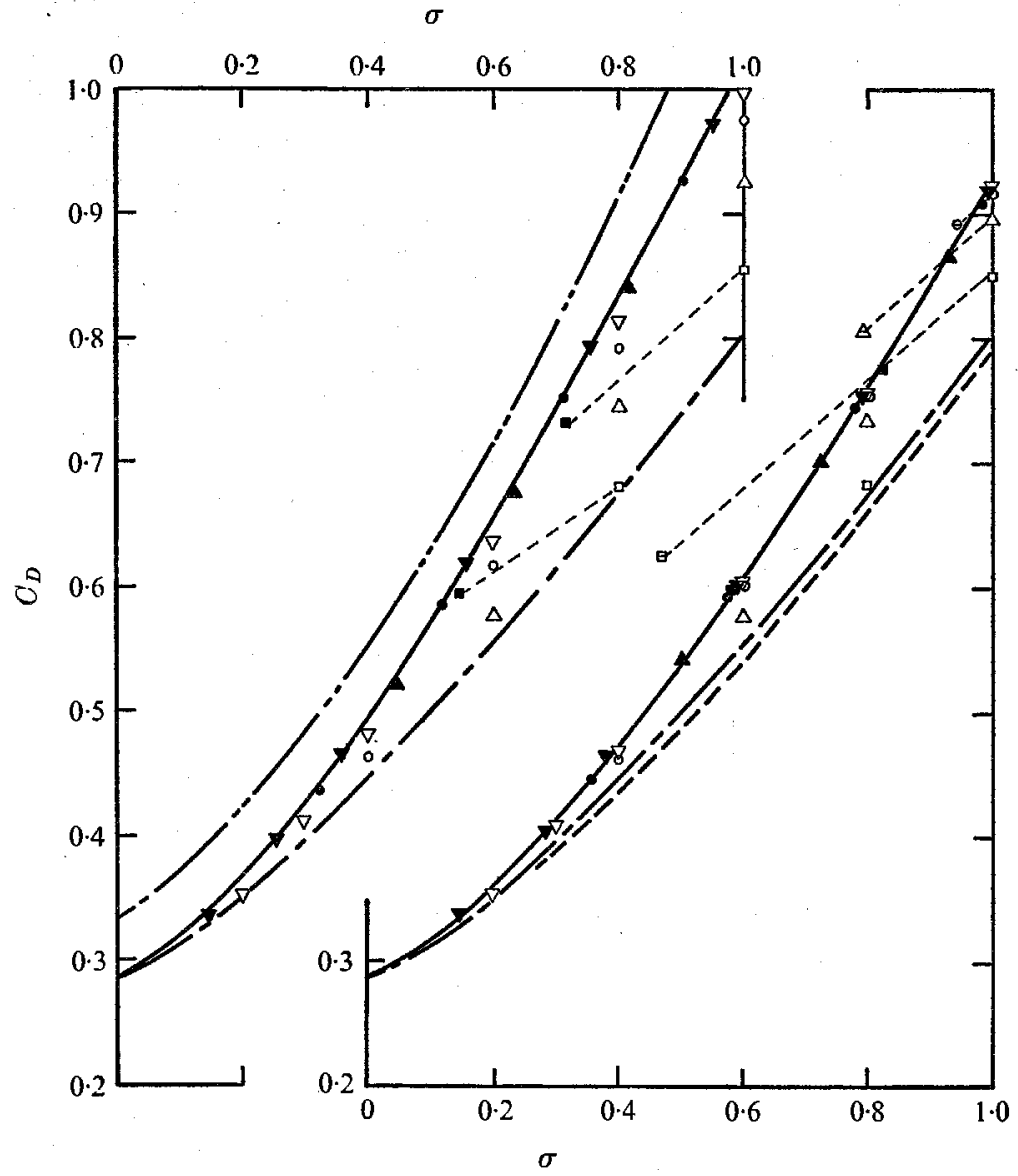

Figure 8. Correction rules tested against theoretical results for $30^{\circ}$ wedge.

$\begin{array}{cccc}\lambda=l / h & \begin{array}{c}\text { Computed } \\ C_{D}(\sigma, \lambda)\end{array} & \begin{array}{c}\text { Corrected to } \\ C_{D}\left(\sigma^{\prime}, 0\right)\end{array} & \begin{array}{c}\text { Meijer's corrected } \\ C_{D}^{\prime \prime}\left(\sigma^{\prime \prime}\right)\end{array} \\ 0.025 & \nabla & \nabla & \\ 0.050 & \bigcirc & \bullet & \ominus \\ 0.100 & \triangle & \Delta & \triangle \\ 0.167 & \square & \bullet & \ominus\end{array}$

Open-wake model (top): ____, choked flow in linearized solution (Cohen \& Gilbert); , unbounded flow $C_{D}(\sigma, 0) ;-\cdots$, , choked flow $C_{D}(\sigma)$. Riabouchinsky model (bottom): unbounded flow; - - , choked flow; - - - , choked flow estimate (see (88)). 
Another important case occurs for small angle wedges $\left(\beta \pi<15^{\circ}\right)$ and $\sigma$ fairly large, when

$$
C_{D}(\sigma, 0) \simeq \sigma
$$

is a good approximation (see figure 4). Then we find

$$
C_{D}(\sigma, \lambda)=C_{D}(\sigma, 0)-\lambda /(\mathrm{I}-\lambda)
$$

which is in excellent agreement with numerical evaluations of the exact solution as well as with the correction rule (77), (78).

\subsection{Riabouchinsky model}

For this model the exact solution (45), (46) shows that $C_{D}(\sigma, \lambda)$ and $\lambda$ are functions of the parameters $a, b$, which depend on the upstream velocity $U$ and the maximum velocity $V$ on the wall according to the same relationships $a=a(U)$, $b=a(V)$, as given by (35), (36) for the open-wake model. In order to examine the rate of change of $b$, as the 'tunnel-spacing ratio' $\lambda$ is varied, and the role played by the minimum pressure $p_{b}$ and the maximum velocity $V$ on the wall (as was once investigated by Meijer 1967, see (6)) we also introduce a new cavitation number $\sigma^{\prime \prime}$ based on $p_{b}$ and $V$ as

$$
\sigma^{\prime \prime}=\left(p_{b}-p_{c}\right) / \frac{1}{2} \rho V^{2}=V^{-2}-1=\sigma(V),
$$

where $\sigma(U)$ gives the conventional cavitation number (see (4)),

$$
\sigma=\sigma(U)=U^{-2}-1 .
$$

The unbounded flow limit $\lambda=0$ is reached as $b \rightarrow a$, which implies $V \rightarrow U$ and $\sigma^{\prime \prime} \rightarrow \sigma$. In order to estimate $C_{D}$ for small $\lambda$, we expand $C_{D}(\sigma, \lambda)$, given by (46) in Taylor's series for $\left|\sigma^{\prime \prime}-\sigma\right| \ll \mathrm{I}$ :

$$
\frac{C_{D}(\sigma, \lambda)}{1+\sigma}=\frac{C_{D}(\sigma, 0)}{1+\sigma}+\left(\sigma^{\prime \prime}-\sigma\right)\left[\frac{\partial}{\partial \sigma^{\prime \prime}}\left(\frac{C_{D}(\sigma, \lambda)}{1+\sigma}\right)\right]_{b=a}+O\left(\sigma^{\prime \prime}-\sigma\right)^{2}
$$

Now, by (46), (36) and (81),

$$
\left[\frac{\partial}{\partial \sigma^{\prime \prime}}\left(\frac{C_{D}(\sigma, \lambda)}{1+\sigma}\right)\right]_{b=a}=-\left[\frac{\partial}{\partial b}\left(\frac{I_{-}(a, b)}{I_{+}(a, b)}\right) \frac{d b}{d V} \frac{d V}{d \sigma^{\prime \prime}}\right]_{b=a} .
$$

Since the functional dependence of $\sigma^{\prime \prime}$ on $b$ is the same as that of $\sigma$ on $a$ (see (35), (36), (81), (82)), we have

$$
\left[\frac{d b}{d V} \frac{d V}{d \sigma^{\prime \prime}}\right]_{b=a}=\frac{d a}{d U} \frac{d U}{d \sigma}
$$

Furthermore, from ( $46 b$ ) it immediately follows that

$$
\left[\frac{\partial}{\partial b} I_{ \pm}(a, b)\right]_{b=a}=\frac{1}{3} \frac{d}{d a} I_{ \pm}(a, a)
$$

Combining these results, we have

$$
\left[\frac{\partial}{\partial \sigma^{\prime \prime}}\left(\frac{C_{D}(\sigma, \lambda)}{1+\sigma}\right)\right]_{b=a}=-\frac{1}{3} \frac{d}{d a}\left(\frac{I_{-}(a, a)}{I_{+}(a, a)}\right) \frac{d a}{d \sigma}=\frac{1}{3} \frac{d}{d \sigma}\left(\frac{C_{D}(\sigma, 0)}{1+\sigma}\right)
$$


Upon substituting (84) in (83), the resulting equation can evidently be written as

$$
\frac{C_{D}(\sigma, \lambda)}{1+\sigma}=\frac{C_{D}\left(\sigma^{\prime}, 0\right)}{1+\sigma^{\prime}}+O\left(\lambda^{2}\right)
$$

where

$$
\sigma^{\prime}=\sigma+\frac{1}{3}\left(\sigma^{\prime \prime}-\sigma\right)=\frac{2}{3} \sigma+\frac{1}{3} \sigma^{\prime \prime},
$$

and $\sigma^{\prime \prime}$, as given by (81) can be obtained either by actual measurement in experiments or it can be calculated if $V$ is known. The parameter $b$ related to $V$ by (36) can be estimated from (45) and (46b) as

$$
b=a+\frac{1}{2 a}\left[\frac{\pi \lambda}{2 U \sin \beta \pi I_{+}(a, a)}\right]^{2}+O\left(\lambda^{2}\right),
$$

so that $V$ can be found from (36). This correction rule has also been compared with the numerical results of the exact solution of $C_{D}(\sigma, \lambda)$; the agreement is again excellent for wedges of all angles with $\lambda<\frac{1}{6}, \sigma<1$. An example is shown in figure 8 for $\beta \pi=15^{\circ}$. Its application in experiments will be discussed in $\S 8$.

In the choked flow limit, $V \rightarrow 1$; hence $\sigma^{\prime \prime} \rightarrow 0$, and $\sigma \rightarrow \sigma_{*}$, say; so that (85) becomes

$$
\frac{C_{D}\left(\sigma_{*}, \lambda\right)}{1+\sigma_{*}}=\frac{C_{D}\left(\frac{2}{3} \sigma_{*}, 0\right)}{1+\frac{2}{3} \sigma_{*}}
$$

This equation gives the choked flow drag coefficient if the unbounded drag coefficient as a function of $\sigma$ is known, or vice versa. As an example of the use of (88), we estimate the choked flow $C_{D}$ for $\beta \pi=15^{\circ}$ in figure 8 and compare this with the computed value.

It is noteworthy that (85) is identical to (77); only $\sigma^{\prime}$ is different in these two theoretically derived wall correction rules. To this end, we note that $\sigma^{\prime}$ in (78b) is known once $\sigma, \lambda$ and $C_{D}(\sigma, \lambda)$ are measured, whereas in (85) and (86), $\sigma^{\prime \prime}$ requires an additional measurement of either $V$ or $p_{b}$.

Another point worthy of note is that, although the significance of $\sigma^{\prime \prime}$ was explored by Meijer (1967), its use in Meijer's empirical rule leads to an overcorrection of the wall effect on drag coefficient, as demonstrated in figure 8 . This is because in Meijer's rule, $\sigma^{\prime \prime}$ takes the place of $\sigma^{\prime}$, instead of a weighted contribution as given by (86).

Finally, we observe that in these two sets of wall correction rules the body configuration has become implicitly absorbed in the drag coefficient as one of its arguments (i.e. $C_{D}(\sigma, \lambda ; \beta)$ ). In view of the result that these correction rules are extremely accurate over the entire range of $\beta(0<\beta<1)$, it is reasonable to expect that they are also valid for bodies of arbitrary shape, at least for those with not too great curvature of their surface profiles.

\section{Experiments}

The first concern of the experimental investigation was the verification of the theoretical analyses and of the correction rules derived from the theory. However, at the same time the opportunity is taken to discuss some of the other problems 
and real fluid effects that arise during cavitation experiments in high-speed water tunnels. These may be grouped as follows:

(i) Viscous effects due to the boundary layer on the model being tested.

(ii) Viscous and other effects due to the boundary layer on the tunnel walls, including production of a longitudinal pressure gradient and acceleration, and the possible appearance of secondary flows.

(iii) The necessity of determining the cavity pressure $p_{c}$; effects which cause this to differ from $p_{v}$, the vapour pressure.

(iv) The determination of a hypothetical 'free stream' pressure $p_{\infty}$ equal to the remote pressure where the tunnel is infinitely long.

( $\nabla)$ Limitations on the range of cavitation number that can be satisfactorily covered including the effect of flow choking.

(vi) Effects due to actual cavity closure. These include the unsteady, turbulent nature of the flow in this region, the cavity filling effect of the re-entrant jet (especially when this impinges on the rear of the headform) and the viscous, turbulent wake behind the cavity.

Some discussion of these problems and effects is included in $§ 8$.

The experiments were carried out in the high-speed water tunnel at the California Institute of Technology. Four wedges of vertex angle $2 \beta \pi=7 \frac{1}{2}{ }^{\circ}, 9^{\circ}$, $15^{\circ}$ and $30^{\circ}$ (chord $\approx 6 \mathrm{in}$.) were tested in the $6 \mathrm{in}$. span, two-dimensional working section (Kiceniuk 1964) of normal height $30 \mathrm{in}$. However, by fitting the tunnel with inserts the $9^{\circ}$ and $30^{\circ}$ wedges were also run with a wall spacing of $13.45 \mathrm{in}$. The models were supported in the centre of the tunnel on a three component force balance for direct measurement of total drag. At the conclusion of each set of experiments the tare drag forces on the fairing plate and wedge supports were measured by replacing that plate by a blank, supporting the wedge in the same position but fastened to the opposite side-wall and measuring the drag registered under conditions identical to those of the main experiments. Subtracting this tare drag from the original drag reading yielded a measure of the force on the wedge alone.

A working section reference pressure, $p_{T}$, was measured at a point in the centre of the side-wall about $7 \mathrm{in}$. upstream of the leading edge of the model using a water/mercury/air manometer (see §8). The hypothetical. 'free stream' velocity in the working section $U$ was inferred from the difference between $p_{T}$ and the pressure upstream of the convergent section. A series of static pressure taps on the lower wall were connected to an inverted water manometer referenced to $p_{T}$ for the purpose of determining the wall pressure distribution. Since some differences were observed even with no model installed in the tunnel, values more representative of the effect of the model were obtained by using these 'clear tunnel' readings as datum.

All four wedges included a base pressure tapping used to measure cavity pressure, $p_{c}$, the technique employed being a familiar one (Brennen 1969a). The pressure line is connected through a two-way push-pull valve to an air supply adjusted so that the air flow keeps the line free of liquid. Activating the valve cut off this supply and connected in an air/mercury/water manometer from which, following an interval of a few seconds, the difference $\left(p_{T}-p_{c}\right)$ could be obtained. 
Two of the wedges, the $9^{\circ}$ and $30^{\circ}$, were built up from the basic model used by Meijer (1967), in order to utilize the static pressure tubes distributed along one face of that model. Fifteen of these were connected to a water/mercury manometer board referred to $p_{T}$ in order to obtain wetted surface pressure distributions; bleeding of these lines before every reading was required to obtain reliable data.

For each model configuration data was obtained over a series of cavitation numbers $\sigma$, at a few selected velocities $U$. However, apart from the limit imposed by flow choking (i.e. $\sigma>\sigma_{*}$ ), there were certain other physical limitations upon the range of $\sigma$ which could be safely and satisfactorily covered at a particular velocity. At higher velocities ( 35 to $50 \mathrm{ftsec}^{-1}$ depending on model size) readings could be obtained only up to a certain $\sigma$, for above this either the drag exceeded that measurable by the balance $(120 \mathrm{lb}$ ) or the vibration of the whole structure became excessive. At lower velocities (25-40 ft sec ${ }^{-1}$ depending on model size) a minimum $\sigma$ was usually imposed by the fact that an excessive number of vapour/ air bubbles appeared in the pressure lines when $p_{T}$ was less than about $0.45 \mathrm{ft}$ of mercury. In the case of the reduced tunnel, vibration of the inserts and oscillation of the flow around them was an added hazard. In general, however, an acceptable range of $\sigma$ could be obtained by combining the results at two velocities, one in the higher range, the other in the lower.

A recurring problem in water tunnel experiments arises in determining a hypothetical, 'free stream' pressure corresponding to the remote pressure, $p_{\infty}$ of potential flow calculations which assume the working section to be infinitely long. In a tunnel of constant section, a favourable longitudinal pressure gradient is produced by boundary-layer growth on the walls. In the present tunnel this could be overcome by flairing the side walls (Kiceniuk 1964). Then the longitudinal pressure gradient is given roughly by

$$
\frac{\partial C_{p}}{\partial x}=\frac{2}{B} \frac{\partial B}{\partial x}-\frac{4(B+h)}{B h} \frac{\partial \delta_{D}}{\partial x}
$$

where $\delta_{D}$ is some mean boundary-layer displacement thickness, $x$ the centre-line distance, and $B(x)$ is the tunnel breadth. Under normal operational conditions the boundary layer is probably turbulent, so that $\partial \delta_{p} / \partial x$ may be given by 0.038 $(\nu / x U)^{\frac{1}{5}}$, though the effective origin of $x$ is difficult to estimate. However, both the experiments of Kiceniuk (1964) and the above formula when, say, $x^{\frac{1}{5}}$ is of order $1 \mathrm{ft}^{\frac{1}{5}}$ and $U$ is between 30 and $50 \mathrm{ft} \mathrm{sec}^{-1}$ indicate that $\partial C_{p} / \partial x$ is roughly zero when $\partial B / \partial x$ is about 0.003 . Thus the flair is set at this value. Nevertheless, since pressures are to be measured on the model itself it seems wise to locate the reference pressure tap as close to the model as possible, yet far enough away for the influence of the pressure field around the model to be negligible. The choice of a tap $7 \mathrm{in}$. from the leading edge of the model involved such compromises. Theoretical estimates indicated that the pressure field influence was less than $\Delta C_{p}=0.01$ at that point. Further upstream the influence of the tunnel convergent section is felt (e.g. 6 in. further upstream, $C_{p}$ was of the order of 0.03 higher). 


\section{Experimental results}

It will be seen that of the theoretical models that of Riabouchinsky yields results closest to the experimental measurements. To avoid confusion by profusion, comparison is made in most of the figures only with that model, whilst comments on the other model will be included in the text. Typical pressure distributions on the faces of the $9^{\circ}$ and $30^{\circ}$ wedges are shown in figures 9 and 10 , where $s$ is measured along the wetted surface from the leading edge, and $s=S$ at separation. These agree quite well with the theory, though two deviations are

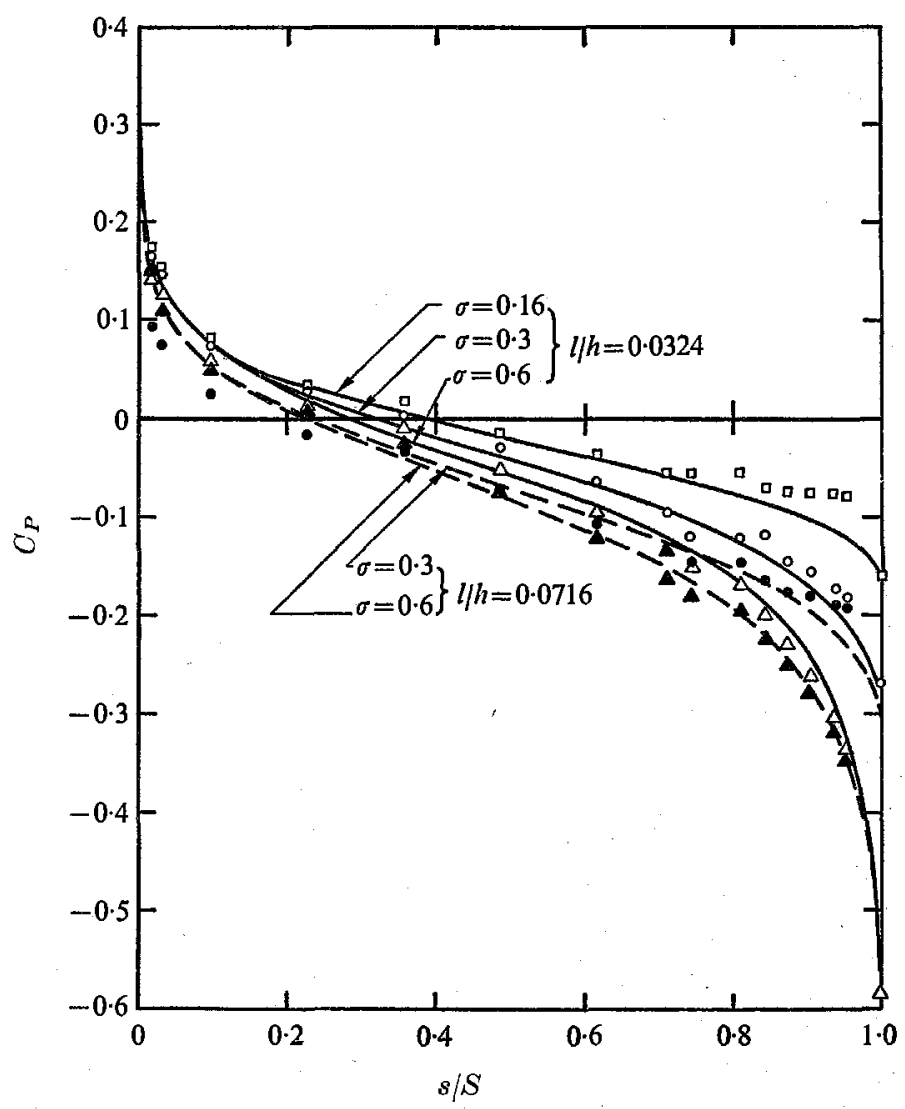

Figure 9. Pressure distribution on the $9^{\circ}$ wedge. Theoretical Riabouchinsky model: ,$- l / h=0.0323 ; \cdots . ., l / h=0.0716$, for $\sigma$ as shown. Experimental points:

$\begin{array}{llllll}l / h & 0.0324 & 0.0324 & 0.0324 & 0.0716 & 0.0716 \\ \sigma & 0.159 & 0.267 & 0.585 & 0.308 & 0.587\end{array}$

noteworthy: (i) the lower experimental $C_{p}$ close to the leading edge are probably due to a slight downward inclination of the incident stream, since small negative lifts were also registered by the balance; (ii) near the trailing edge the experimental $C_{p}$ are slightly above the theory, especially when the flow is close to being choked. This second effect may be partly due to the presence of small air/vapour bubbles 
in the tubes registering these low pressures, though there may also be some contribution from the complex boundary-layer flow near separation. Note also how the effect of the wall is to depress the centre portion of the pressure distribution while the end points $\left(C_{p}=1\right.$ at stagnation, $=-\sigma$ at separation $)$ remain unchanged.

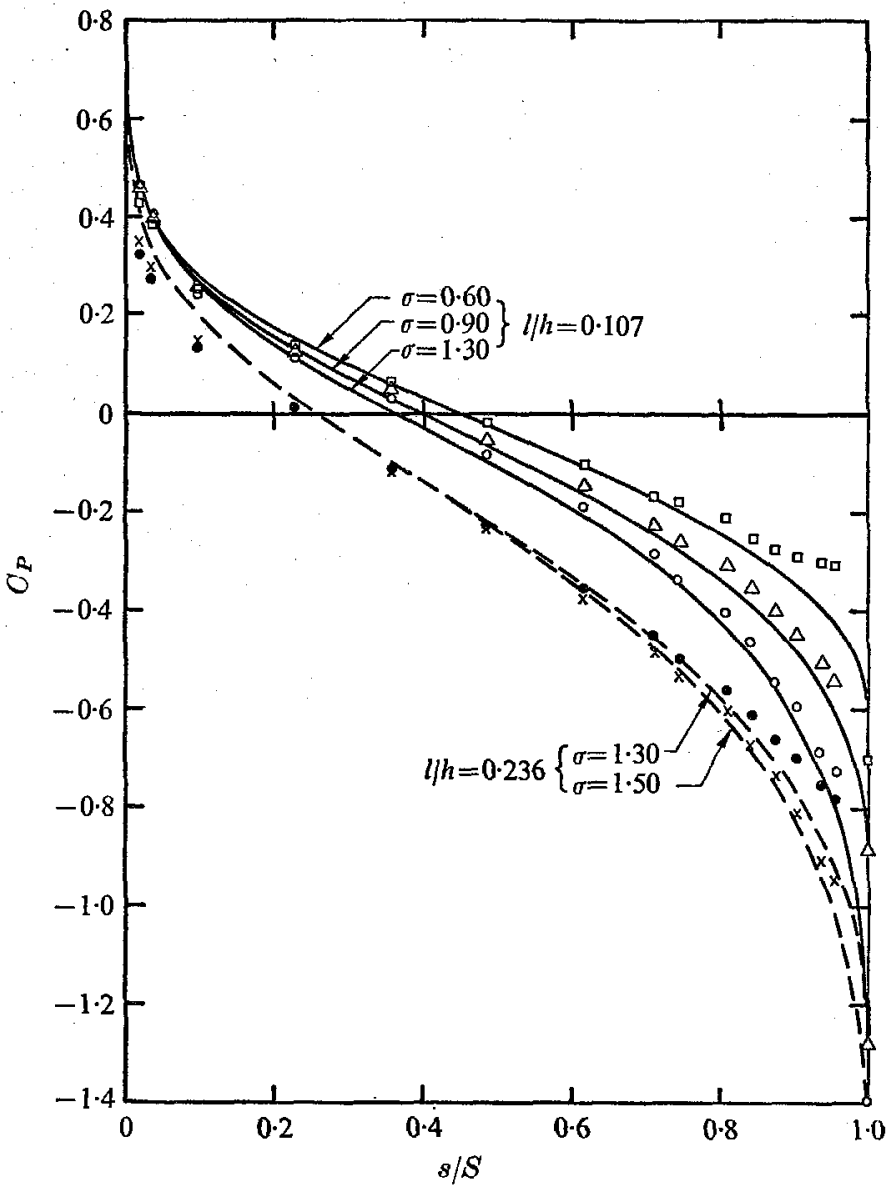

Frgure 10. Pressure distribution on the $30^{\circ}$ wedge. Theoretical Riabouchinsky model: ,$- l / h=0 \cdot 107 ; \cdots, l / h=0 \cdot 236$, for $\sigma$ as shown. Experimental points:

$\begin{array}{lccccc}l / h & 0 \cdot 107 & 0 \cdot 107 & 0 \cdot 107 & 0 \cdot 236 & 0.236 \\ \sigma & 0 \cdot 60 & 0.882 & 1 \cdot 393 & 1.28 & 1.54 \\ & \square & \triangle & 0 & - & \times\end{array}$

Coefficients of drag are plotted in figures 11 and 12. Graphic integration of the experimental pressure distributions yields results in excellent agreement with the Riabouchinsky model theory. The direct measurements, corrected for tare drag, showed a greater scatter and the comparison is poorer. An estimate of the skin friction component of this total drag was obtained using the Faulkner-Skan solutions for the boundary-layer flow near the leading edge of a wedge. Then

$$
\left(\Delta C_{D}\right\rangle_{\text {viscous }}=\frac{2(2(n+1))^{\frac{1}{2}}}{3 n+1} \frac{A^{\frac{3}{4}}}{\tan \beta \pi} f^{\prime \prime}(0)\left(\frac{\nu}{S U}\right)^{\frac{1}{2}},
$$


where $n=\beta /(1-\beta), A$ represents the strength of the leading edge singularity which is estimated from the value of $\left(1-C_{p}\right)(S / s)^{n}$ near that point and takes a value of about unity. In the conventional notation, $f^{\prime \prime}(0)$ is a known function of $\beta$ available in tables of Faulkner-Skan solutions. The work of Ackerberg (1970) would indicate that the contribution of the rapidly accelerating flow near the trailing edge is small in comparison. Equation (90) yields respective values of 0.012 and 0.006 for the $9^{\circ}$ and $30^{\circ}$ wedge experiments, and these are included in the figures, with, as can be seen, mixed results.

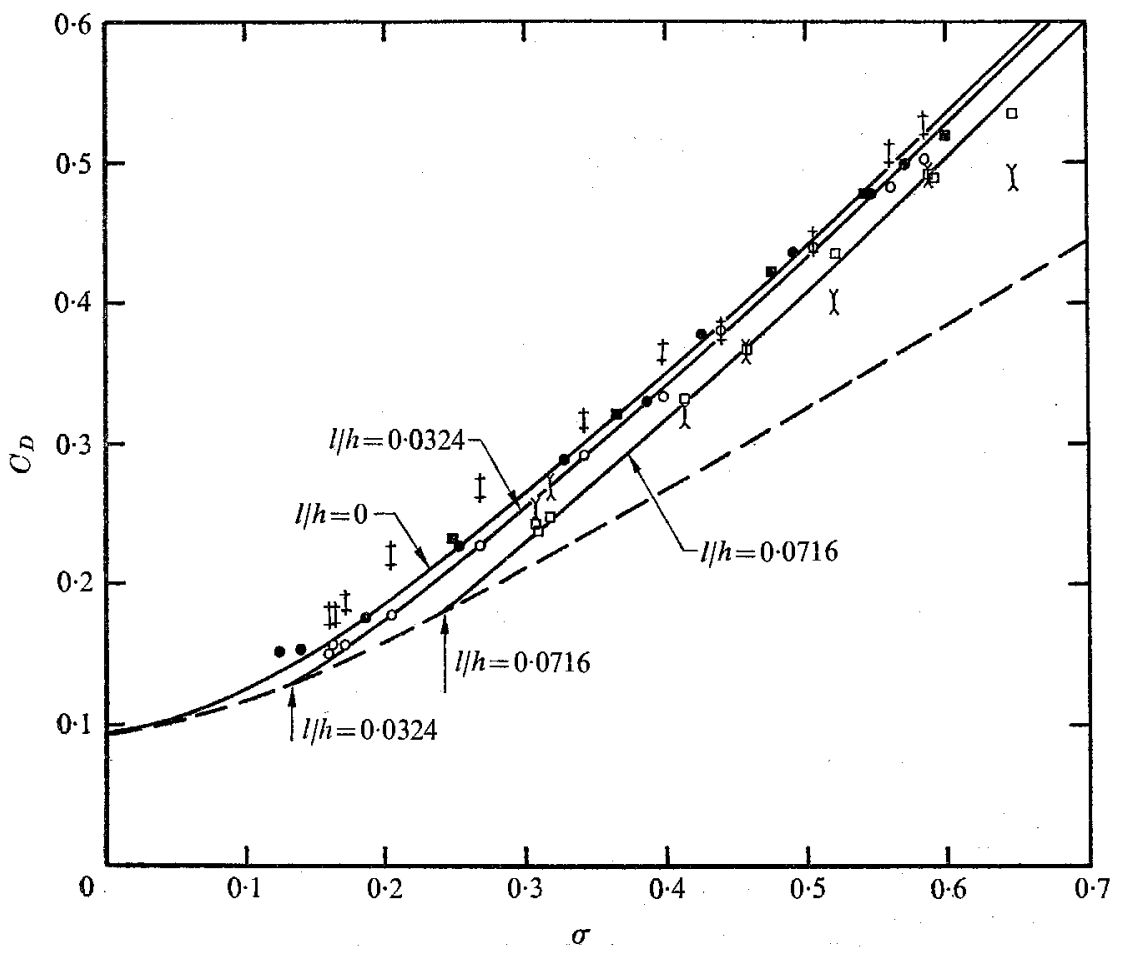

Figure 1I. Drag of the $9^{\circ}$ wedge $\left(\beta \pi=4 \cdot 5^{\circ}\right)$. Theory: —_, Riabouchinsky model; . . . ., choked flow line; $\uparrow$, choked flow at specified $l / h$. Experiment: pressure integration, $\square, l / h=0.0716 ; O, l / h=0.0324 ;$ and $O$, corresponding values corrected to unbounded flow using (85), (86); balance measurement and with viscous drag estimate subtracted, $x, l / h=0 \cdot 0716 ;$ f $l / h=0 \cdot 0324$.

The more reliable data (namely, the pressure-integrated drag coefficients) could also be compared with the results of the open-wake theoretical model. However, it is clear, from the agreement with the Riabouchinsky model and the difference between the two theoretical models (see e.g. figures 5 and 8 ), that the experimental values will lie significantly below the open-wake theory, except close to the choked condition, where the theories virtually coincide in any case. The difference would be especially marked for small $l / h$ at moderate to high $\sigma$. Comparison could also be made with the results of the linearized theory of Cohen \& Gilbert (1957). As expected, the linearized theory yields values of $C_{D}$ substantially greater than either the exact theory or the experiments. This is 
exemplified in figure 8, where it is seen that the linearized theoretical choked flow line is actually above the unbounded flow line for a $30^{\circ}$ wedge. The difference is of course less for wedges of smaller $\beta \pi$.

Previous experimental measurements by Waid (1957) in a solid wall tunnel of $\lambda=0.027$, and by Cox \& Clayden (1958) in a free jet tunnel ( $l /$ free jet width $\approx 0 \cdot 055$ ), are also shown in figure 12 . The wall effect is expected to be small in both instances. Agreement with theory is good, and Waid's measurements indicate a small wall effect of the expected proportions.

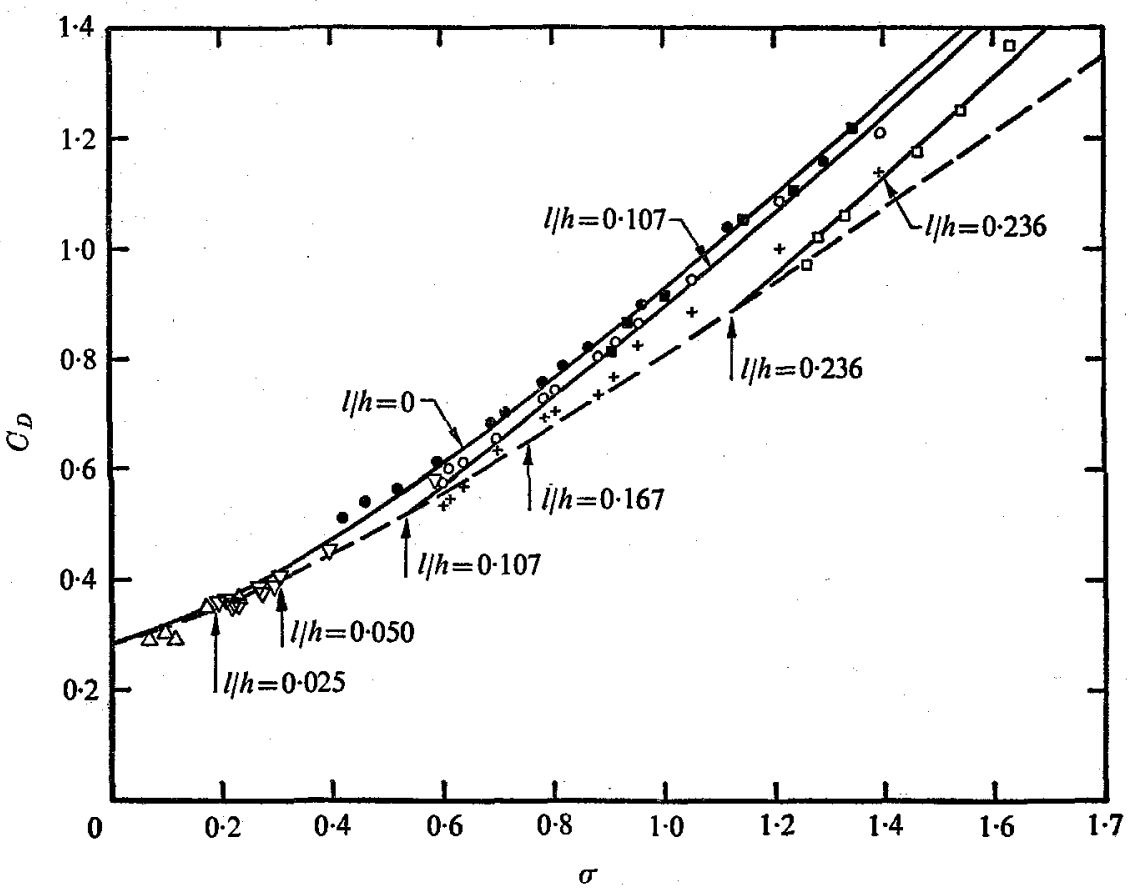

FIGURE 12. Drag of the $30^{\circ}$ wedge $\left(\beta \pi=15^{\circ}\right)$. Theory: $\longrightarrow$, Riabouchinsky model; ...., choked flow line; $\uparrow$, choked flow at specified $l / h$. Experiment: pressure integration, $\square$, $l / h=0.236 ; \bigcirc, l / h=0 \cdot 107$; and $\quad$, corresponding values corrected to unbounded flow using (85), (86); balance measurement,,$+ l / h=0.107$ (viscous estimate, $\Delta C_{D}=0.006$ ). $\nabla$, Experiments of Waid (1957), $l / h=0.027 ; \triangle$, of Cox \& Clayden (1958) in free jet tunnel.

Sample wall pressure distributions, referenced to clear tunnel values as mentioned in $\S 6$, are presented in figure 13 for the case of the $9^{\circ}$ wedge. Note that the cavity wake causes the experimental curves to asymptote to a non-zero $C_{p}$ down-stream of the cavity. Thus, the actual curves correspond to a compromise on the Riabouchinsky model theory in the direction of the open-wake model (the curves for which are not shown but decrease monotonically toward a value corresponding to velocity $V$ ). This deviation clearly causes a slight reduction of the minimum wall pressure below the Riabouchinsky model value. This occurred consistently as can be seen from figure 14 where the minimum wall pressures for all model configurations are plotted against $\sigma$. Nevertheless, the agreement with theory is satisfactory. 
Distance downstream from point opposite trailing edge of model (in.)

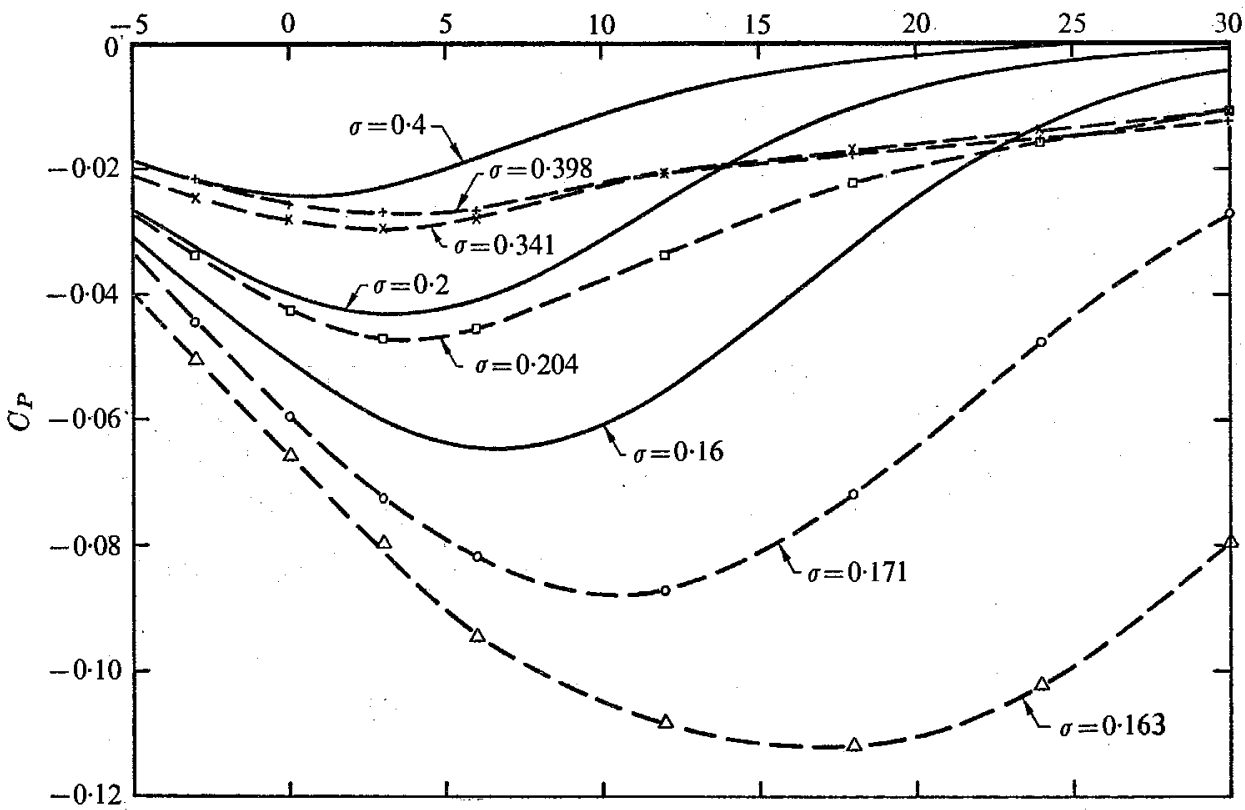

FIaURE 13. Sample wall pressure distributions for $2 \beta \pi=9^{\circ}, l / h=0.0324 . \ldots$, experimental curves; ——, Riabouchinsky model theory.
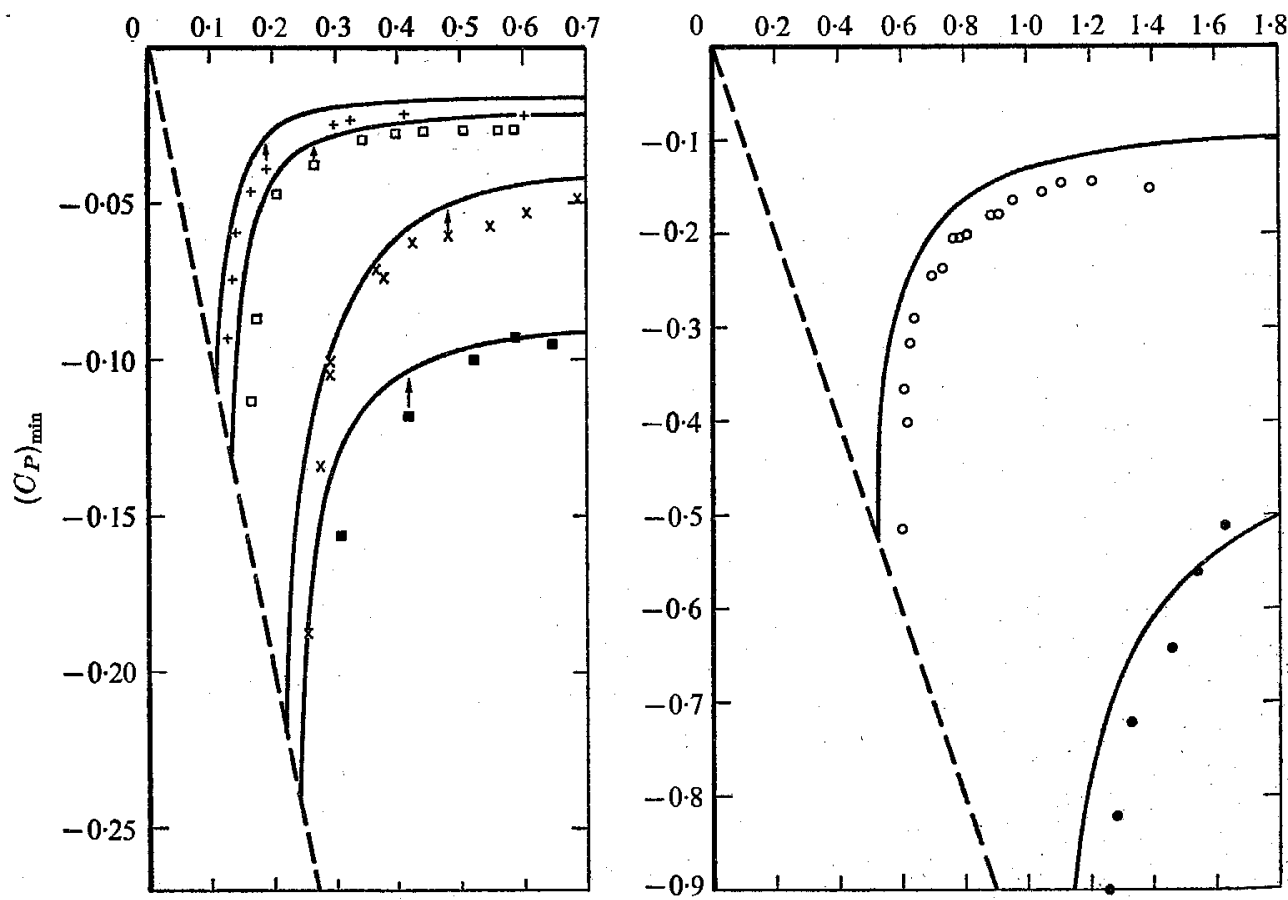

FigURE 14. Minimum wall pressure vs. cavitation number. Experimental points:

$\begin{array}{lcccccc}\text { wedge angle } & 7 \cdot 5^{\circ} & 9^{\circ} & 9^{\circ} & 15^{\circ} & 30^{\circ} & 30^{\circ} \\ l / h & 0.0262 & 0.0324 & 0.0716 & 0.0527 & 0.107 & 0.236 \\ & + & \square & \square & \times & 0 & 0\end{array}$

Corresponding Riabouchinsky model theoretical curves $(-)$ are indicated by arrows; ....., choked flow. 
The pressure-integrated drag on the $9^{\circ}$ and $30^{\circ}$ wedges are corrected for wall effect using the relations (85), (86) and the experimental values of minimum wall pressure. The results are the solid points in figures 11 and 12 . Clearly the results are very satisfactory, since the rule collapses the points for different $l / h$ onto a single line very close to the unbounded theoretical line. The only noticeable deviation is at low $\sigma$, where the experimental points lie somewhat above that theoretical curve.

The authors have pleasure in expressing their appreciation and gratitude to Professor Francis Clauser for invaluable encouragement and to the Division of Engineering and Applied Science of the California Institute of Technology for supporting entirely the experimental investigation using the High-Speed Water Tunnel facility of the Hydrodynamics Laboratory.

The early part of the main theoretical study was carried out under the support of the Naval Ship System Command General Hydrodynamics Research Program and Hydrofoil Advanced Development Program, administered by the Naval Ship Research and Development Center, and the latter part under the support of the Office of Naval Research.

\section{REFERENCES}

ACKerberg, R. C. 1970 Boundary layer separation at a free streamline. Proc. 2nd Int. Conf. on Numerical Methods in Fluid Mechanics, Berkeley, Calif.

AI, D. K. 1966 The wall effect in cavity flows. Trans. ASME, J. Basic Eing. 88D, 132.

Alren, H. J. \& VINCENTI, W. G. 1944 Wall interference in a two-dimensional-flow wind tunnel with consideration of the effect of compressibility. $N A C A T R 782$.

Armstrong, A. H. \& TAdman, K. G. 1953 Wall corrections to axially symmetric cavities in circular tunnels and jets. Ministry of Supply A.R.W. Rep. 7-52.

BARR, R.A. 1966 An investigation of the cavity flow behind drag dises and supercavitating propellers. M.S. Thesis, University of Maryland.

BirkноFғ, G. 1950 Hydrodynamics. Princeton University Press.

Brrkhoft, G., Plesset, M. \& Stmmons, N. 1950 Wall effects in cavity flow I. Quart. Appl. Math. 8, 161.

Btrkнoft, G., Plesset, M. \& Simmons, N. 1952 Wall effects in cavity flow II. Quart. Appl. Math. 9, 413.

Birkioff, G. \& Zarantonello, E. H. 1957 Jets, Wakes and Cavities. Academic.

Brennen, C. $1969 a$ Dynamic balances of dissolved air and heat in natura] cavity flows. J. Fluid Mech. 37, 115.

Brennein, C. $1969 b$ A numerical solution of axisymmetric cavity flows. J. Fluid Mech. 37,671 .

Campbell, I. J. \& Thomas, G. E. 1956 Water tunnel boundary effects on axially symmetric fully developed cavities. Admiralty Res. Lab. R1-G-HY-18-1.

CisotTi, U. 1922 Idromeccanica Piana. Milan.

Cohen, H. \& DiPrima, R. C. 1958 Wall effects in cavitating flows. Proc. 2nd Symp. on Naval Hydrodynamics, ACR-38. Washington, D.C.: Govt. Printing Office.

CoHen, H. \& Gmbers, R. 1957 Two-dimensional, steady, eavity flow about slender bodies in channels of finite breadth. J. Appl. Mech. 24, 170.

Cohen, H., Sutherland, C. C. \& To, Y. 1957 Wall effects in cavitating hydrofoil flow. J. Ship Res. 3, 31.

Cox, A. D. \& Cliayden, W. A. 1958 Cavitating flow about a wedge at incidence. J. Fluid Mech. 3, 615. 
Dоват, G. V. 1967 Experimental investigation of wall effect on simple cavity flows. Proc. Symp. Testing Techniques in Ship Cavitation Research 1, 175. Skipsmodelltanken, Trondheim, Norway.

EpPLER, R. 1954 Beitrage zu Theorie und Anwendung der unstetigen Stromungen. $\dot{J}$. Rat. Mech. Anal. 3, 591.

FABUlA, A. G. 1964 Choked flow about vented or cavitating hydrofoils. Trans. ASME, J. Basic Eng. 86D, 561 .

Gilbarg, D. 1949 Proc. Nat. Acad. Sci. U.S.A. 35, 609.

Gilbarg, D. 1961 Jets and cavities. Handbuch der Physik, 9, 311. Springer.

Gladert, H. 1933 Wind tunnel interference on wing, bodies, and air-screws. $R \& M$. 1566.

Grove, A. S., Shair, F. H., Petersen, E. E. \& Acrivos, A. 1964 An experimental investigation of the steady separated flow past a circular cylinder. J. Fluid Mech. 19, 60 .

GUREvion, M. I. 1953 Proc. A. I. Mikoyan, Moscow Techn. Inst. of Fis. Ind. Econ. 5.

JoukowskY, N. E. 1890 A modification of Kirchhoff's method of determining a two dimensional motion of a fluid given a constant velocity along an unknown streamline. Rec. Math. 25. (Also 1936 Collected Works of N. E. Joukowsky 3, 195. Moscow).

KICENIUK, T. 1964 A two-dimensional working section for the high speed water tunnel at the California Institute of Technology. ASME, Cav. Res. Fac. Tech.

MeiJer, M. C. 1967 Pressure measurement on flapped hydrofoils in cavity flows and wake flows. J. Ship. Res 11, 170.

MeiJer, M. C. 1969 Discussion of the Cavitation Committee Rep., 12th Int. Towing Tank Conf., Rome.

Morgan, W.B. 1966 The testing of hydrofoils and propellers for fully-cavitating or ventilated operation. Proc. 11th Int. Towing Tank Conf., Tokyo.

PoPE, A. 1958 Wind Tunnel Testing (2nd edn.). Wiley.

Roshko, A. 1954 A new hodograph for free streamline theory. NACA TN 3168.

Simmons, N. 1948 The geometry of liquid cavities with especial reference to the effects of finite extent of the stream. Ministry of Supply, A.D.E. Rep. 17-48.

VAtcovicr, V. 1913 Über discontinuierliche Flussigkeitsbewegungen mit zwei freien Strahlen. Thesis, Göttingen University.

VILLAT, H. 1914 Sur la validite des solutions de certains problemes d'hydrodynamique. J. Math. (6) 10, 231.

WAID, R. L. 1957 Water tunnel investigation of two-dimensional cavities. Calif. Inst. of Tech. Rep. E-73.6.

WANG, D. P. \& WU, T. Y. 1963 Small-time behaviour of unsteady cavity flows. Arch. Rat. Mech. Anal. 14, 127.

WHTINEY, A. K. 1969 A simple correction rule for wall effect in two-dimensional cavity flow. Cavitation: State of Knowledge. New York: ASME.

Wu, T. Y. 1962 A wake model for free streamline flow theory. Part 1. Fully and partially developed wake flows and cavity flows past an oblique flat plate. J. Fluid Mech. 13, 161.

Wu, T. Y. 1968 Inviscid cavity and wake flows. Basic Developments in Fluid Dynamics (ed. M. Holt). Academic.

WU, T. Y. \& WANG, D. P. $1964 a$ A wake model for free-streamline flow theory. Part 2. Cavity flows past obstacles of arbitrary profile. J. Fluid Mech. 18, 65.

Wv, T. Y. \& WANG, D. P. $1964 b$ An approximate numerical scheme for the theory of cavity flows past obstacles of arbitrary profile. Trans. ASME, J. Basic Eng. 86D, 556.

Wб, T. Y., WhItNeY, A. K. \& LIN, J. D. 1969 Wall effect in cavity flows. Calif. Inst. of Tech. Rep. E-111A. 5. 